\title{
() Assessment of Adult Women With Ovarian Masses and Treatment of Epithelial Ovarian Cancer: ASCO Resource-Stratified Guideline
}

Verna D. Vanderpuye, MD ${ }^{1}$; Jean Rene V. Clemenceau, MD²; Sarah Temin, MSPH³ Zeba Aziz, MD; William M. Burke, MD ${ }^{5}$; Nixon Leonardo Cevallos, MD ; Linus T. Chuang, MD; Terence J. Colgan, MD ${ }^{8}$; Marcela G. del Carmen, MD ${ }^{9}$; Keiichi Fujiwara, MD, PhD ${ }^{10}$; Elise C. Kohn, MD ${ }^{11}$; Jose Enrique Gonzáles Nogales, MD ${ }^{12}$; Thomas Okpoti Konney, MD ${ }^{13}$; Asima Mukhopadhyay, MD, PhD, MSc ${ }^{14,15}$; Bishnu D. Paudel, MD ${ }^{16}$; Icó Tóth ${ }^{17}$; Sarikapan Wilailak, MD ${ }^{18}$; and Rahel G. Ghebre, MD, MPH ${ }^{19,20}$

PURPOSE To provide expert guidance to clinicians and policymakers in three resource-constrained settings on diagnosis and staging of adult women with ovarian masses and treatment of patients with epithelial ovarian (including fallopian tube and primary peritoneal) cancer.

METHODS A multidisciplinary, multinational ASCO Expert Panel reviewed existing guidelines, conducted a modified ADAPTE process, and conducted a formal consensus process with additional experts.

RESULTS Existing sets of guidelines from eight guideline developers were found and reviewed for resourceconstrained settings; adapted recommendations from nine guidelines form the evidence base, informing two rounds of formal consensus; and all recommendations received $\geq 75 \%$ agreement.

RECOMMENDATIONS Evaluation of adult symptomatic women in all settings includes symptom assessment, family history, and ultrasound and cancer antigen 125 serum tumor marker levels where feasible. In limited and enhanced settings, additional imaging may be requested. Diagnosis, staging, and/or treatment involves surgery. Presurgical workup of every suspected ovarian cancer requires a metastatic workup. Only trained clinicians with logistical support should perform surgical staging; treatment requires histologic confirmation; surgical goal is staging disease and performing complete cytoreduction to no gross residual disease. In first-line therapy, platinum-based chemotherapy is recommended; in advanced stages, patients may receive neoadjuvant chemotherapy. After neoadjuvant chemotherapy, all patients should be evaluated for interval debulking surgery. Targeted therapy is not recommended in basic or limited settings. Specialized interventions are resourcedependent, for example, laparoscopy, fertility-sparing surgery, genetic testing, and targeted therapy. Multidisciplinary cancer care and palliative care should be offered.

ASSOCIATED

CONTENT

Appendix

Data Supplement

Author affiliations

and support

information (if

applicable) appear at

the end of this

article.

Accepted on May 17, 2021 and published at ascopubs.org/journal/ go on June 29, 2021: Dol https://doi.org/10. $1200 /$ G0.21.00085

Practice Guidelines Committee approval: February 24, 2021 Reprint Requests: 2318 Mill Road, Suite 800 , Alexandria, VA 22314; guidelines@ asco.org

Additional information can be found at www.asco.org/resource-stratified-guidelines. It is ASCO's view that health care providers and health care system decision makers should be guided by the recommendations for the highest stratum of resources available. The guideline is intended to complement but not replace local guidelines.

JCO Global Oncol 7:1032-1066. ๑ 2021 by American Society of Clinical Oncology

Creative Commons Attribution Non-Commercial No Derivatives 4.0 License (c) () ()

\section{INTRODUCTION}

The purpose of this guideline is to provide expert guidance on the diagnosis and treatment of adult women 18 years of age or older with epithelial ovarian cancer (EOC) (including fallopian tube and primary peritoneal cancer) to clinicians, public health leaders, patients, and policymakers in resource-constrained settings. The target population is adult women with ovarian masses and other symptoms of ovarian cancer as well as those diagnosed with EOC at all stages in resource-constrained settings. This guideline is not intended for patients in maximal settings, as described in Table 3.

Ovarian cancer is often diagnosed at an advanced stage, stage III or IV. All women are at risk for ovarian cancer; women with genetic predisposition; personal or family history of breast, ovarian, or colon cancer; infertility; and advancing age are at higher than population-based risk. There is currently no reliable screening method or primary prevention available for ovarian cancer in any setting. Therefore, most women with ovarian cancer are diagnosed on the basis of symptomatic presentation with the majority at advanced stages across all resource settings. In basic settings, chest x-ray and abdominal ultrasound are typically the only imaging modalities available. Women with ovarian cancer report nonspecific symptoms that may be overlooked or misdiagnosed by primary care providers and contribute to delay in diagnosis. In resource-constrained settings, patients with advanced 


\section{THE BOTTOM LINE}

\section{Assessment of Adult Women With Ovarian Masses and Treatment of Epithelial Ovarian Cancer: ASCO Resource-Stratified Guideline Key Questions}

For each of the three resource-constrained settings levels (basic, limited, and enhanced):

(A) What are the optimal diagnosis and staging strategies for adult women with ovarian masses and/or EOC (including fallopian tube and primary peritoneal cancer)?

(B) What is the optimal surgery for women with stages I-IV EOC (including fallopian tube and primary peritoneal cancer)?

(C) What is the optimal adjuvant and/or systemic therapy for stages I-IV EOC (including fallopian tube and primary peritoneal cancer)?

(D) What is the optimal therapy for women with recurrent EOC (including fallopian tube and primary peritoneal cancer)?

\section{Target Population}

Adult women (18 years of age or older) in three resource-constrained settings levels with ovarian masses and/or diagnosed with EOC (including fallopian tube and primary peritoneal cancer)?

\section{Target Audience}

This guideline globally targets health care providers (including gynecologic oncologists, medical oncologists, radiation oncologists, obstetricians and gynecologists, surgeons, nurses, and palliative care clinicians) and nonmedical community members, including patients, caregivers, and member(s) of advocacy groups.

\section{Methods}

A multinational, multidisciplinary Expert Panel was convened to develop clinical practice guideline recommendations on the basis of a systematic review of the medical literature and an expert consensus process.

Author's note: It is the view of the ASCO that health care providers and health care system decision makers should be guided by the recommendations for the highest stratum of resources available. The guidelines are intended to complement but not replace local guidelines. General statement about recommendations: referral to higher-resource level settings, if feasible, is preferable.

\section{Key Recommendations-Because of the Large Number of Recommendations, Only a Summary of Key Recommendations Are in This Box}

\section{Clinical question $A$.}

What are the optimal diagnostic strategies for adult women with ovarian masses and/or symptoms of EOC (including fallopian tube and primary peritoneal cancer)?

- General practitioners should perform a clinical assessment and family history and where available, aid diagnosis by ultrasound (abdominal and transvaginal ultrasound, Doppler-enhanced) AND/OR contrast-enhanced computed tomography (CT) of abdomen and pelvis (with or without thorax).

- In postmenopausal women with symptoms of ovarian cancer, cancer antigen 125 [CA-125] value can assist in diagnosis.

- Ovarian cancer is diagnosed with histologic confirmation in all settings.

- CT-guided biopsy or laparoscopy (with sufficient resources) is preferred instead of laparotomy to obtain histologic confirmation prior to any systemic therapy.

See Table 5 and Appendix Figures A1 and A7.*

\section{Clinical question $B$.}

What is the optimal surgery for women with stages I-IV EOC (including fallopian tube and primary peritoneal cancer)?

- The purpose of surgery is to diagnose, to stage, and/or for treatment.

- Ovarian cancer surgery should be performed by trained gynecologic oncologists or surgeons with oncology surgical expertise. Refer patients to highest-resourced level oncology center with oncology surgical capacity.

- Staging: Where feasible, patients with presumed early-stage ovarian cancer should undergo surgical staging by trained surgeon(s). In basic settings, surgical staging is not feasible, thus not recommended.

- Treatment: Women with advanced ovarian cancer (stage III and IV) should receive optimal surgical debulking to remove all visible disease to improve overall survival (OS) by trained surgeon(s).

See Tables 5 and 6 and Appendix Figures A2, A8, and A9.*

General statement about chemotherapy: Access to appropriate evidence-based chemotherapy agents, contraindications to chemotherapy, and potential side effects of chemotherapy should be evaluated and managed in every patient. Basic-resource

(continued on following page) 


\section{THE BOTTOM LINE (CONTINUED)}

settings that most likely lack the capacity to provide safe administration of chemotherapy should refer patients to a higher-level center for evaluation. Limited settings without skilled capacity should refer patients to settings with access to specialized care.

\section{Clinical question $\boldsymbol{C}$.}

What is the optimal adjuvant and/or systemic therapy for stages I-IV EOC (including fallopian tube and primary peritoneal cancer)?

- Clinicians should document pathology and stage to determine eligibility for adjuvant chemotherapy. If pathology confirmation is not possible because of patient or resource limitation, alternatives can be discussed.

- Clinicians should not administer (systemic treatment) adjuvant chemotherapy to patients with ovarian low-malignant potential tumors or early-stage microinvasive borderline tumors, independent of stage.

- Combination chemotherapy with paclitaxel and carboplatin is the standard of care for adjuvant therapy in ovarian cancer.

- Single-agent carboplatin may be used because of resource limitation or patient characteristics.

- Only in enhanced settings, highly selected cases can be assessed for appropriate evidence-based intraperitoneal (IP) chemotherapy, following optimal debulking, where there are resources and expertise to manage toxicities.

See Table 6 and Appendix Figures A3, A4, and A10.*

\section{Clinical question $D$.}

What is the optimal treatment for women with recurrent EOC (including fallopian tube and primary peritoneal cancer)?

- For recurrent disease in limited or enhanced settings only, patients with recurrent ovarian cancer should be counseled on treatment options on the basis of a patient's prior response to platinum-based chemotherapy, that is, platinum-sensitive, platinum-resistant, or platinum-refractory disease status. Platinum rechallenge is only recommended for patients with platinum-sensitive disease.

- In enhanced settings only, clinicians may offer maintenance systemic therapies.

- Treatment is not recommended for patients with tumor marker-positive (CA-125) only recurrent ovarian cancer.

- Early palliative care interventions benefit all patients diagnosed with ovarian cancer.

- See related ASCO guidelines in the Appendix.

See Table 7 and Appendix Figures A5, A6, and A10.*

General statement about heritable risk: For women with strong family history of breast and/or ovarian cancer, clinicians should discuss family history and refer to counseling or testing, if available.

\section{Additional Resources}

More information, including a supplement, slide sets, and clinical tools and resources, is available at www.asco.org/resourcestratified-guidelines. The Methodology Manual (available at www.asco.org/guideline-methodology) provides additional information about the methods used to develop this guideline. Patient information is available at www.cancer.net.

\section{ASCO believes that cancer clinical trials are vital to inform medical decisions and improve cancer care and that all patients should have the opportunity to participate.}

*Full list of recommendations and corresponding tables are available in the Data Supplement.

ovarian cancer may not seek care or undergo diagnosis; therefore, some deaths may not be recorded in hospital registries. Underreporting may be a contributing factor to the low numbers recorded in countries with low Human Development Index (HDI) (Table 1). The HDI refers to a composite measure of development, not limited to income alone. ${ }^{1}$

Pathology is an important part of diagnosis and guides the management of women with EOC. The Expert Panel recognizes that there is variable availability and financing for the development of pathology services around the world. This document further discusses pathology in the Special Commentary section.

Mortality-to-incidence ratios for EOC are very high, more than $65 \%$ per American Cancer Society figures within the
United States. It is notable that there is a histologic difference and an incidence difference across racial and ethnic populations. Clear cell histology (which can be found in up to $30 \%$ of early-stage cases) is two to four times as prevalent in Japan as elsewhere. EOC, in general, is less common in Africans for unknown reasons, but may be attributable to underreporting. In 2020, GLOBOCAN presented its data in terms of the $\mathrm{HDI}$, rather than focusing on countries by income level. The terms often overlap, but not entirely, with the terms low- and middle-income countries. The Bray et al analysis of these data reported, in Figure 7 , incidence and mortality age-standardized rates in high-HDI plus very high-HDI regions versus low-HDI plus medium$\mathrm{HDI}$ regions. Both rates were higher in the former (7 high plus very high HDI v 5.7 low plus medium HDI (incidence); 
TABLE 1. Incidence and Mortality of Ovarian Cancer

HDI

No.

Crude Rate ASR

\begin{tabular}{lrrr}
\hline Incidence of ovarian cancer & & & \\
\hline All & 313,959 & 8.1 & 6.6 \\
\hline Low & 15,379 & 3.1 & 5.1 \\
\hline Medium & $6,559,480,973$ & 5.8 & 6.0 \\
\hline $\begin{array}{l}\text { Combined low HDI, low income, low } \\
\text { middle income, medium HDI }\end{array}$ & 176,709 & 5.2 & 5.9 \\
\hline High & 116,505 & 8.1 & 6.1 \\
\hline Very high & 116,347 & 14.7 & 8.3 \\
\hline Mortality of ovarian cancer & & & \\
\hline All & 207,252 & 5.4 & 4.2 \\
\hline Low & 11,106 & 2.2 & 4.0 \\
\hline Medium & $4,559,856,704$ & 4.0 & 4.3 \\
\hline Combined low HDI, low income, low & 122,452 & 3.6 & 4.2 \\
$\quad$ middle income, medium HDI & & & \\
\hline High & 76,796 & 5.3 & 3.8 \\
\hline Very high & 73,655 & 9.3 & 4.3
\end{tabular}

Abbreviations: ASR, age-standardized rate; HDI, Human Development Index.

${ }^{a}$ Crude and ASRs per 100,000 per Cancer Today (International Agency for

Research on Cancer, WHO). World Health Organization: International Agency for

Research on Cancer: Cancer Today. https://gco.iarc.fr/today/home

3.8 high plus very high $v 4$ low plus medium [mortality]). Data are from the Cancer, American Cancer Society table, new cases, and deaths for 36 cancers and all cancers combined in 2018, page $398^{2}$ and here.

However, mortality-to-incidence ratios are high in all regions, notably highest in low- to medium-HDI regions. Data regarding women from Africa and women of African descent in the United States demonstrate the highest mortality rates, attributable to less-resourced health systems. ${ }^{3}$ As of 2018 , data showed $27 \%$ of deaths from ovarian cancer occur in low- and medium-HDI regions, whereas $25 \%$ of the estimated 295,414 new cases occur in these regions around the world. GLOBOCAN data show highest incidence and mortality rates were in high-HDI plus very high-HDI regions (Table 1) (Source: GLOBOCAN 2020). In some so-called more-developed regions, rates are decreasing. ${ }^{4}$

Different regions of the world, both among and within countries, have variable access to diagnosis and treatment of EOC. Patients with cancer of the ovary ideally require the care of specialized surgical teams including gynecologic oncologists and general surgeons who have extensive training in oncology. However, outside of specialized centers within high-HDI regions, there is a paucity of specialty training with few clinicians available to skillfully manage these patients. ${ }^{5}$ Some of the presumptions inherent in the guideline include that chemotherapy and specialized surgery are not available in basic settings (Table 2). As a result of these disparities, the American Society of Clinical Oncology (ASCO) Resource-Stratified Guidelines Advisory Group chose epithelial cancer of the ovary as a priority topic for guideline development.

ASCO has established a process for development of resource-stratified guidelines, ${ }^{6}$ which includes mixed methods of evidence-based guideline development, adaptation of the clinical practice guidelines of other organizations, and formal expert consensus. This article summarizes the results of that process and presents resource-stratified recommendations (see Results section).

TABLE 2. Diagnosis/Staging/Treatment Capacities by Setting

\begin{tabular}{|c|c|c|c|}
\hline Intervention & Basic & Limited & Enhanced \\
\hline Imaging & X-ray and US and expertise for interpretation & $\begin{array}{l}\text { X-ray/US/CT may be available in some } \\
\text { regions } \\
\mathrm{CT} \text {-guided/US-guided biopsy available }\end{array}$ & CT/MRI available \\
\hline Surgery & $\begin{array}{l}\text { General practitioner with basic surgical } \\
\text { capacity (can include some ovarian mass } \\
\text { diagnostic procedures-not hysterectomy) } \\
\text { General surgery facility with minor OR available } \\
\text { with anesthesia }\end{array}$ & $\begin{array}{l}\text { General surgeon, general surgery facility } \\
\text { with OR, Ob/Gyn—by default has } \\
\text { some oncology skills }\end{array}$ & $\begin{array}{l}\text { OR, ICU, most major surgeries available, } \\
\text { subspecialized oncologists, including } \\
\text { surgical oncologists/gynecologic } \\
\text { oncologists }\end{array}$ \\
\hline Chemotherapy & $\begin{array}{l}\text { Presume not available (for purposes of } \\
\text { guidelines) } \\
\text { Availability of chemotherapy drugs is } \\
\text { unpredictable }\end{array}$ & $\begin{array}{l}\text { Some chemotherapy available. } \\
\text { Only first-line }\end{array}$ & $\begin{array}{l}\text { More chemotherapy options available, } \\
\text { targeted therapy may or may not be } \\
\text { available. May be } \geq \text { first-line available }\end{array}$ \\
\hline Pathology & $\begin{array}{l}\text { Sending pathology for review when needed } \\
\text { may or may not be available }\end{array}$ & $\begin{array}{l}\text { Pathology services in development, } \mathrm{H \& E} \\
\text { usually available, IHC and molecular } \\
\text { tests are usually not available }\end{array}$ & $\begin{array}{l}\text { Pathology services usually available and } \mathrm{IHC} \\
\text { and molecular tests may be available }\end{array}$ \\
\hline
\end{tabular}

Abbreviations: CT, computed tomography; H\&E, hematoxylin and eosin stain; ICU, intensive care unit; IHC, immunohistochemistry; MRI, magnetic resonance imaging; Ob/Gyn, obstetrician/gynecologist; OR, operating room; US, ultrasound. 
In developing resource-stratified guidelines, ASCO has adopted its framework from the four-tier resource setting approach (basic, limited, enhanced, and maximal; Table 3) developed by Breast Health Global Initiative and modifications to that framework on the basis of the Disease Control Priorities 3.,8 The framework emphasizes that variations occur not only between but also within countries with disparities, for example, between rural and urban areas.

\section{GUIDELINE QUESTIONS}

This clinical practice guideline addresses the following four overarching clinical questions:

(A) What are the optimal diagnosis and staging strategies for adult women with ovarian masses and/or EOC (including fallopian tube and primary peritoneal cancer)?

(B) What is the optimal surgery for women with stages I-IV EOC (including fallopian tube and primary peritoneal cancer)?

(C) What is the optimal adjuvant and/or systemic therapy for stages I-IV EOC (including fallopian tube and primary peritoneal cancer)?

(D) What is the optimal therapy for women with recurrent EOC (including fallopian tube and primary peritoneal cancer)?

TABLE 3. Framework of Resource Stratification

Setting

Basic

Core resources or fundamental services that are absolutely necessary for any public health/primary health care system to function; basic-level services typically are applied in a single clinical interaction. Vaccination is feasible for highest-need populations

Limited

Second-tier resources or services that are intended to produce major improvements in outcome such as incidence and cost-effectiveness and are attainable with limited financial means and modest infrastructure; limitedlevel services may involve single or multiple interactions. Universal public health interventions feasible for greater percentage of population than primary target group

Enhanced

Third-tier resources or services that are optional but important; enhanced-level resources should produce further improvements in outcome and increase the number and quality of options and individual choice (perhaps ability to track patients and links to registries)

Maximal

May use high-resource settings' guidelines

High-level/state-of-the-art resources or services that may be used/available in some high-resource countries and/or may be recommended by highresource setting guidelines that do not adapt to resource constraints but that nonetheless should be considered a lower priority than those resources or services listed in the other categories on the basis of extreme cost and/or impracticality for broad use in a resource-limited environment

NOTE. Data adapted. ${ }^{7,8}$ To be useful, maximal-level resources typically depend on the existence and functionality of all lower-level resources. Maximal-level recommendations are not included in this guideline.

\section{METHODS}

\section{Guideline Development Process}

This systematic review-based guideline product was developed by an multinational, multidisciplinary Expert Panel, which included a patient representative and ASCO guidelines staff member with health research methodology expertise (Appendix Table A2). The Expert Panel met via teleconference and corresponded through e-mail. On the basis of the consideration of the evidence, the authors were asked to contribute to the development of the guideline, provide critical review, and finalize the guideline recommendations. Members of the Expert Panel were responsible for reviewing and approving the penultimate version of the guideline, which was then circulated for external review and submitted to a peer-reviewed journal for editorial review and consideration for publication.

This guideline adaptation was also informed by the ADAPTE methodology and consensus methodology together as an alternative to de novo guideline development for this guideline. Adaptation of guidelines is considered by ASCO in selected circumstances when one or more quality guidelines from other organizations already exist on the same topic. The objective of the ADAPTE process ${ }^{9}$ is to take advantage of existing guidelines to enhance efficient production, reduce duplication, and promote the local uptake of quality guideline recommendations.

ASCO's adaptation process begins with a literature search by ASCO guidelines staff, to identify candidate guidelines for adaptation. Adapted guideline manuscripts are reviewed and approved by the ASCO Clinical Practice Guidelines Committee (CPGC). The review includes two parts: methodologic review and content review. The methodologic review is completed by a member of the CPGC's Methodology Subcommittee and/or by ASCO guidelines staff. The content review is completed by an Expert Panel (Appendix Table A2). All funding for the administration of the project was provided by ASCO. Further details of the methods used for the development of this guideline are reported in the ASCO Guidelines Methodology Manual (available at www.asco.org/guideline-methodology).

This guideline was partially informed by ASCO's modified Delphi Formal Expert Consensus methodology, during which the Expert Panel was supplemented by additional experts recruited to rate their agreement with the drafted recommendations. The entire membership of experts is referred to as the Consensus Panel (a list of members is available in Appendix Table A3). In round 1, 20 experts (plus two who were on the Expert Panel) participated; in round 2, there were a total of 26 respondents (nine of whom were on the Expert Panel). The guideline recommendations were crafted, in part, using the Guidelines Into Decision Support methodology. ${ }^{10}$ The guideline recommendations were sent for an open comment period of 2 weeks allowing the public to review and comment on the recommendations 
after submitting a confidentiality agreement. These comments were taken into consideration while finalizing the guideline. All ASCO guidelines are ultimately reviewed and approved by the Expert Panel and the ASCO Clinical Practice Guideline Committee prior to publication.

\section{Guideline Disclaimer}

The clinical practice guidelines and other guidance published herein are provided by the ASCO to assist providers in clinical decision making. The information therein should not be relied upon as being complete or accurate, nor should it be considered as inclusive of all proper treatments or methods of care or as a statement of the standard of care. With the rapid development of scientific knowledge, new evidence may emerge between the time information is developed and when it is published or read. The information is not continually updated and may not reflect the most recent evidence. The information addresses only the topics specifically identified therein and is not applicable to other interventions, diseases, or stages of diseases. This information does not mandate any particular course of medical care. Further, the information is not intended to substitute for the independent professional judgment of the treating provider, as the information does not account for individual variation among patients. Recommendations reflect high, moderate, or low confidence that the recommendation reflects the net effect of a given course of action. The use of words like "must," "must not," "should," and "should not" indicate that a course of action is recommended or not recommended for either most or many patients, but there is latitude for the treating physician to select other courses of action in individual cases. In all cases, the selected course of action should be considered by the treating provider in the context of treating the individual patient. Use of the information is voluntary. ASCO provides this information on an "as is" basis, and makes no warranty, express or implied, regarding the information. ASCO specifically disclaims any warranties of merchantability or fitness for a particular use or purpose. ASCO assumes no responsibility for any injury or damage to persons or property arising out of or related to any use of this information or for any errors or omissions.

\section{Guideline and Conflicts of Interest}

The Expert Panel was assembled in accordance with ASCO's Conflict of Interest Policy Implementation for Clinical Practice Guidelines ("Policy," found at http://www. asco.org/rwc). All members of the Expert Panel completed ASCO's disclosure form, which requires disclosure of financial and other interests, including relationships with commercial entities that are reasonably likely to experience direct regulatory or commercial impact as a result of promulgation of the guideline. Categories for disclosure include employment; leadership; stock or other ownership; honoraria, consulting or advisory role; speaker's bureau; research funding; patents, royalties, other intellectual property; expert testimony; travel, accommodations, expenses; and other relationships. In accordance with the Policy, the majority of the members of the Expert Panel did not disclose any relationships constituting a conflict under the Policy.

\section{RESULTS}

\section{Literature Search}

The recommendations were developed through a systematic review of high-quality published guidelines and clinical experience. A search for new evidence was conducted by ASCO guidelines staff to identify systematic review-based guidelines published between January 2012 and March 2019 in PubMed, Cochrane Systematic Reviews, US AHRQ database (the formerly extant) and US National Guideline Clearinghouse databases and complemented with searches of G-I-N International Guideline Library $^{11}$ (see the Data Supplement for details on the search). The search was restricted to articles published in English, French, or Spanish. Guidelines were selected for inclusion in the systematic review on the basis of the following criteria:

1. addressed the diagnosis or treatment of ovarian masses and/or ovarian cancer,

2. developed by multidisciplinary content experts as part of a recognized organizational effort, and

3. published between 2012 and 2019 (later narrowed to 2014-2019 to capture more current information).

The Expert Panel suggested two additional guidelines for review. The Expert Panel later narrowed the date parameter to between January 2014 and December 2019 (with the addition of some Panel-suggested literature and ASCO guidelines published up to June 2020).

Articles were excluded from the systematic review if they were (1) meeting abstracts; (2) books, editorials, commentaries, letters, news articles, case reports, or narrative reviews; or (3) primary literature. After initial searches of primary literature, the panel leadership decided to primarily use guidelines to inform expert consensus. ASCO considered quality guidelines that either met the US National Guidelines Clearinghouse 2013 criteria as assessed by National Guideline Clearinghouse or met ASCO criteria for Appraisal of Guidelines for Research and Evaluation II (AGREE II) methodologic review. Searches for cost-effectiveness analyses were also conducted separately.

A total of 156 titles of guidelines were found in the literature searches. The ASCO Expert Panel reviewed nine of the guidelines that met inclusion criteria, in-depth for their currency, content, and methodology. On the basis of content and methodology reviews, the Expert Panel chose six non-ASCO guidelines and three ASCO guidelines (Scottish Intercollegiate Guidelines Network [SIGN], ${ }^{12}$ Belgian Health Care Knowledge Centre $[\mathrm{KCE}],{ }^{13}$ ASCO and Society of Gynecologic Oncology [SGO], ${ }^{14,15}$ Ontario 
Health-Cancer Care Ontario [OH-CCO], ${ }^{16}$ Japan Society of Gynecologic Oncology [JSGO], ${ }^{17}$ British Gynaecological Cancer Society [BGCS], ${ }^{18}$ Irish National Clinical Effectiveness Committee [NCEC], ${ }^{19}$ and the 2020 ASCO guidelines ${ }^{20,21}$ ). These evidence-based guidelines were developed by eight health authorities and/or guideline developers (SIGN, KCE, ASCO, SGO, OH-CCO, JSGO, BGCS, and Irish NCEC; one was a joint ASCO and SGO guideline). Appendix Table A1 lists links to the guidelines. The Expert Panel used these guidelines, literature suggested by the Expert Panel, and clinical experience as guides. The Expert Panel formally vetted the included guidelines' content and development methodology. The Data Supplement encompasses a detailed overview of the included guidelines, including information on the clinical questions, target populations, development methodology, and key evidence.

This ASCO guideline reinforces selected recommendations offered in the SIGN, Belgian KCE, ASCO and SGO, ASCO, $\mathrm{OH}-\mathrm{CCO}, \mathrm{JSGO}, \mathrm{BGCS}$, and Irish NCEC guidelines and acknowledges the effort put forth by the authors and aforementioned societies to produce evidence-based and/ or consensus-based guidelines informing practitioners and institutions who provide care to patients with ovarian masses and/or ovarian cancer.

\section{SUMMARY OF ADAPTED GUIDELINES}

\section{Guidelines on Assessment of Ovarian Masses and Treatment of Patients Diagnosed With Epithelial Ovarian Cancer}

The Expert Panel identified clinical questions and/or categories within the adapted guidelines that would potentially match the ASCO clinical questions. All the guidelines were developed on the basis of patients in maximal settings; therefore, the Expert Panel had to review and adapt the recommendations for resource-constrained settings on the basis of experience in resource-constrained settings and then validate the recommendations by formal consensus. ${ }^{22}$ All the guideline developers used different methods. Most of the maximal setting guidelines had clinical questions or key questions, including the Belgian KCE, Irish NCEC, ASCO and SGO, all the ASCO guidelines, JSGO, OH-CCO, and SIGN guidelines; the BGCS guidelines did not explicitly label clinical questions. The target populations were all in maximal settings and included people with ovarian masses, suspected ovarian cancer, and/or patients with carcinoma of the ovary, fallopian tube carcinoma, and primary peritoneal carcinoma - primarily epithelial ovarian carcinoma and are coalesced for the purposes of this document as epithelial ovarian cancer (EOC). The Irish NCEC guideline's target population is specifically people with ovarian masses/ suspected ovarian cancer, and the ASCO Germline and Somatic Tumor Testing guideline ${ }^{20}$ focuses on women diagnosed with ovarian cancer (with one recommendation on first- or second-degree blood relatives of a patient with ovarian cancer with a known germline pathogenic cancer alteration; however, that discussion is outside the scope of this resource-stratified guideline). Four of the guidelines included both diagnosis and treatment: including Belgian KCE, BGCS, ASCO and SGO, and JSGO guidelines. Three, including the ASCO guideline on poly (ADP-ribose) polymerase inhibitors (PARPi), $\mathrm{OH}-\mathrm{CCO}$ guidelines $(\mathrm{OH}-\mathrm{CCO}$ was specifically on patients with EOC recurrence), and JSGO guidelines, focused on treatment only. Since this ASCO resource-stratified guideline does not include patients with germ cell tumors, sections of adapted guidelines that targeted that population were not used. Specific clinical questions (if provided) and target populations of the adapted guidelines are listed in the Data Supplement.

At the time of the systematic searches for high-quality existing guidelines for this ASCO resource-stratified guideline, there were multiple existing guidelines from maximal settings (see the Data Supplement). Four of the non-ASCO guidelines and all the adapted ASCO guidelines (including the guideline of ASCO and SGO) used systematic review-based methods. Two of the guidelines found were not traditionally systematic review-based. The key evidence the guidelines used included systematic reviews, meta-analyses, nonsystematic literature reviews, existing guidelines, observational studies, and consensus. Most of the evidence regarded systemic therapy. In some areas regarding other interventions, the guidelines used observational data. Therefore, many recommendations in this ASCO guideline were informed by this variety of expertreviewed data and then validated by Formal Consensus.

The outcomes or end points in most studies reviewed by the adapted guidelines included efficacy (including overall survival and progression-free survival [PFS]), quality of life (QoL), safety and/or adverse events, and in some cases, cost-effectiveness.

\section{RESULTS OF ASCO METHODOLOGIC REVIEW}

The methodologic review of the guidelines was completed by two ASCO guideline staff members for each guideline using the Rigour of Development subscale of the AGREE II instrument (with the exceptions of the guidelines that ASCO developed [neoadjuvant, testing, and PARPi]). The score for the Rigour of Development domain is calculated by summing the scores across individual items in the domain and standardizing the total score as a proportion of the maximum possible score. Detailed results of the scoring and the AGREE II assessment process for this guideline are available in the Data Supplement.

\section{SELECTED RECOMMENDATIONS}

The recommendations were developed by a multinational, multidisciplinary group of experts using evidence from existing guidelines and clinical experience as a guide. The ASCO Expert Panel underscores that health care practitioners who implement the recommendations presented in this guideline should first identify the available resources in 
their local and referral facilities and endeavor to provide the highest level of care possible with those resources. The authors would like to make some general points applying to recommendations throughout this guideline: outcomes should be balanced with QoL including financial toxicity; recommendations are made regarding what is feasible in resource-constrained settings.

Because of the large breadth of recommendations, the Panel elected to discuss selected areas.

\section{OVERARCHING CLINICAL QUESTION A}

What are the optimal diagnosis and staging strategies for adult women with ovarian masses and/or EOC?

\section{Evaluation and diagnosis of adult women with ovarian masses or symptoms of EOC (Recommendations 1.1-1.4)}

Recommendations on evaluation and diagnosis for women with ovarian masses are provided in Tables 5 and 6 and Appendix Figures A1, A2, A8, and A9. These recommendations are adapted, and in some cases modified from the guidelines from the developers Belgian KCE, Irish NCEC, SIGN, BGCS, and ASCO and SGO and informed by clinical expertise. ${ }^{23}$

\section{Diagnostic Strategies}

Discussion. These recommendations concern assessment for adult women with ovarian masses in basic, limited, and enhanced settings.

Basic-resource settings. Women with ovarian cancer may report generalized symptoms of pain, fatigue, loss of appetite, abdominal bloating, or feeling full with small meals or early satiety. Other focal signs can include a patient's report of a mass noted in the abdomen or symptoms of abdominal distension, abdominal or pelvic pain, and change in bowel function with diarrhea or constipation (for the latter, symptoms of $<12$ months duration and occurring more than 12 times per month). Women presenting with symptoms associated with possible EOC require an evaluation. The cause of underlying symptoms, generally nonspecific but potentially severe or life-impacting in nature, needs to be determined and may lead to a potential cancer diagnosis. A general practitioner in a basic-resource setting has to rely on the patient's history and physical examination findings to determine the need for diagnostic testing. Initial assessment by a general practitioner includes a complete physical examination, focusing on the abdominal and pelvic examination, to determine the presence of any pelvic or ovarian mass. A clinical diagnosis of ovarian cancer can be discussed on the basis of certain complements of symptoms. A symptom index has been validated and may be helpful in guiding care. ${ }^{24}$ Measuring the serum CA-125 tumor marker alone is not validated for diagnostic use; however, if more than $300 \mathrm{IU} / \mathrm{mL}$, can be suggestive of serous ovarian cancer. CA-125 is less useful for women who are premenopausal, with early-stage ovarian cancer, with ovarian cancer of other epithelial types, or with nonepithelial ovarian tumors.

\section{Imaging}

Women who are postmenopausal with recurrent and persistent symptoms, even in the setting of a negative physical examination, require further evaluation with pelvic ultrasound (transabdominal and transvaginal ultrasound) and referral to a higher-level center for further evaluation including a CT scan. Upon completion of the history and physical examination, where resources allow, ultrasoundbased imaging including a pelvic ultrasound is a general first step in the diagnostic evaluation.

The diagnostic evaluation for an ovarian mass is most widely performed with a pelvic ultrasound. Prospective studies from the International Ovarian Tumor Analysis group have identified 10 characteristics of benign versus malignant ovarian mass that are highly accurate and reproducible for diagnosis of ovarian cancer (Table 4). ${ }^{19}$ Evaluation of these features via pelvic ultrasound with the designation of benign, malignant, or inconclusive, in addition to clinical assessment, provides a general practitioner guidance for a referral to treatment.

A mass is classified as probable malignant if at least one malignant feature and none of the benign features are present and vice versa. If no benign or malignant features are present or if both benign and malignant features are present, then the rules are considered inconclusive (unclassifiable mass), and clinicians should use further clinical and diagnostic testing.

The clinical presentation and imaging findings of both benign (eg, peritoneal tuberculosis) and other malignancies (eg, Gl cancer) may be similar or mimic those of ovarian cancer. Patients in basic-resource settings, when feasible, should be referred to a higher-level care center with capacity for surgical and medical management of patients when there is a concern for malignancy, either of ovarian or other types. Consequently, a histopathologic diagnosis should be undertaken prior to definitive treatment, with referral for diagnosis if feasible. Histology or cytology diagnosis of EOC should be made by a certified pathologist. See the Special Commentary on pathology for further details.

(Sources: SIGN, BGCS, Irish NCEC)

Limited-resource settings. Clinicians should perform the recommendations from the basic setting and may add CT if available. CT imaging of the abdomen and pelvis to include the lower lung fields, using oral and intravenous (IV) contrast where available, can help document the extent of disease spread to solid organ structures such as the liver, detail the severity of peritoneal carcinomatosis, rule out pleural effusion, and aid clinicians in surgical treatment planning (see recommendation 1.4 and recommendations 2.1.1-2.2.3). In women with respiratory symptoms, CT of 
TABLE 4. International Ovarian Tumor Analysis Simple Rules

\begin{tabular}{ll}
\hline Unilocular tumor (B1) & Irregular solid tumor (M1) \\
\hline Largest diameter of largest solid component $<7 \mathrm{~mm}$ (B2) & Ascites (M2) \\
\hline Acoustic shadows (B3) & At least four papillary projections (M3) \\
\hline Smooth multilocular tumor with largest diameter $<100 \mathrm{~mm}$ (B4) & Irregular multilocular solid tumor with largest diameter $\geq 100 \mathrm{~mm}$ (M4) \\
\hline No intratumoral blood flow at color or power Doppler (B5) & Very strong intratumoral blood flow at color or power Doppler (M5)
\end{tabular}

NOTE. Irish National Clinical Effectiveness Committee; Belgian Health Care Knowledge Centre ( $p$ 23).

the thorax provides a more complete evaluation, although this guideline does not recommend routinely performing it for all patients with suspected ovarian cancer in limitedresource settings. A chest $\mathrm{x}$-ray is more accessible and cost-effective for evaluation of the thorax.

(Sources: SIGN, ASCO/SGO, Irish NCEC)

Enhanced-resource settings. In addition to recommendations from the basic and limited settings, magnetic resonance imaging provides a minimal added benefit to the assessment of an ovarian mass suspicious for diagnosis of ovarian cancer. CT imaging of the abdomen, pelvis, and thorax provides a more comprehensive evaluation of disease burden and is also beneficial in surgical treatment planning. Specific benign ovarian pathology such as fibroid disease or dermoid cyst(s) are optimally visualized with ultrasound and thus magnetic resonance imaging of the pelvis can be used only if the clinician's decision making will be altered on the basis of radiologic findings.

(Sources: SIGN, ASCO/SGO, Irish NCEC)

\section{Assessing heritable risk (Recommendation 1.3)}

Discussion. This guideline is not focused on screening and/or genetic tests (the prespecified population in this guideline does not include asymptomatic individuals; ASCO has a maximal setting guideline for testing for women with a personal history of ovarian cancer but acknowledges that there is no global resource to inform what is available, accessible, and paid for by population-level care in each country and/or region).$^{20}$ Assessing heritable risk of ovarian cancer is a part of diagnosis, but the overall population for this umbrella group of recommendations is women with ovarian masses, independent of potential individual heritable risk, and does not include populations that are asymptomatic. During the diagnosis for women with ovarian masses, the guideline stresses that the most important riskrelated intervention is taking family history and related counseling, in all settings, recognizing that genetic counseling is not accessible to most women outside of maximalresource settings. A 2015 ASCO policy statement "affirms that the recognition and management of individuals at inherited risk for cancer is a core element of oncology practice. The skills required to provide cancer risk assessment services are not specific to a discipline but rather incorporate elements from oncology, medical genetics and genetic counseling, and other disciplines. ASCO recommends continued education of oncologists and other health care professionals in the area of cancer risk assessment and management of individuals with an inherited predisposition to cancer." 25

Family history includes the patient's age, cancer history, childbearing status and preferences, and that of close relatives (especially first- and second-degree relatives). First-degree relatives include a patient's mother, father, sister, brother, daughter, or son; second-degree relatives are the first-degree relative(s) of a patient's first-degree relatives (grandparents, grandchildren, parent(s') siblings and their children, and half-siblings). Recommendations on genetic testing and how to best manage results are still equivocal in both resource-rich and resource-constrained settings.

Basic-resource settings. Clinicians evaluating women for ovarian masses in basic-resource settings should obtain a comprehensive family cancer history. Recognizing the heritable risk of ovarian cancer, family history of ovarian cancer are key supportive data to guide diagnosis. Families of women with a diagnosis of ovarian cancer seeking genetic counseling should be referred to a higher-level center with clinicians trained in cancer risk management.

Limited-resource and enhanced-resource settings. Clinicians should be mindful that to offer genetic testing, actionable next steps should be available, for example, follow-up counseling and genetic marker-based treatment(s). Guidelines serving as this resource-stratified guideline's evidence base, for example, SIGN, state with a low level of evidence that screening for ovarian cancer in high-risk groups without confirmed diagnosis of personal or family history of cancer should only be offered in the context of a research study. ${ }^{12}$ For additional reading, refer to evidence-based guidelines that explore the harms and benefits of BRCA testing and other genetic testing for individuals and populations at high risk, see Appendix Table A4.

(Source: informal consensus on the basis of Expert Panel opinion)

\section{Minimally invasive techniques (Recommendation 1.4)}

Discussion. Patients should be referred from basic or limited settings to higher-resourced settings wherever 
feasible for diagnostic procedures. Minimally invasive biopsy procedures may include paracentesis and thoracocentesis for cytology or needle tissue biopsy for pathology. Specificity of diagnosis on the basis of cytology alone varies on the basis of histology type. Cytology can be nonspecific and sometimes does not exclude other nonovarian primary cancers but paired with high CA-125 levels in women who are postmenopausal can be indicative of high-grade serous ovarian cancer. An image-guided intraabdominal tissue biopsy can be obtained where there is established clinical capacity. Laparoscopy can be used to obtain tissue for histologic diagnosis and assessment of the extent of disease for determination of whether to use neoadjuvant chemotherapy (NACT) in limited or enhanced settings. When histologic-based diagnosis cannot be obtained, a CA 125 to carcinoembryonic antigen ratio $>25$ can support ovarian cancer as the primary diagnosis. Clinicians with laparoscopic training in oncology surgical care may use laparoscopy for limited indications.

(Sources: BGCS/Irish NCEC/ASCO/SGO)

\section{OVERARCHING CLINICAL QUESTION B}

What is the optimal surgery for women with stages I-IV EOC?

\section{Staging for suspected stage I/II ovarian cancer (Recommendation 2.1.1)}

Recommendations on staging are in Table 5, Appendix Figures A2 and A8, and the Data Supplement.

Discussion. Surgery is an essential element in ovarian cancer diagnosis and initial care, necessary for accurate diagnosis of ovarian cancer. Surgical staging follows or coincides with diagnostic interventions. Invasive intervention in patients with ovarian cancer is done for three reasons: tissue pathology diagnosis, surgical staging, or tumor debulking with the goal to achieve optimal tumor cytoreduction to RO (no gross visible disease). In apparent early-stage ovarian cancer (stage I/II), appropriate surgical management of the ovarian mass includes minimizing risk of rupture. Surgical staging in apparent early-stage ovarian cancer provides prognostic information through comprehensive staging, if available. Complete staging helps to define prognosis and may change treatment course if a patient's disease is unexpectedly upstaged and should ideally be undertaken by a subspecialized gynecologic oncology surgeon with appropriate experience or, where limitations exist, a gynecologist or general surgeon experienced in pelvic surgery. Surgery is performed to stage and remove all visible tumors; this may involve more than one surgical procedure or more than one surgical specialist to accomplish. Because of the complexity of ovarian cancer surgery and perioperative management of patients with ovarian cancer, patients should be referred to the highestlevel care center with the capacity for expert surgical and medical management.

Surgical staging involves assessment and biopsies of the pelvis and abdomen, en bloc resection of the fallopian tubes, ovaries, uterus (see the Data Supplement for options in fertility-sparing surgery), infracolic omentectomy, evaluation of the bowel serosa and peritoneal surfaces from infradiaphragmatic space to the floor of the pelvis, pericolic gutter washings, and evaluation of the inferior aspect of the diaphragm. Systematic pelvic and para-aortic lymph node dissection is controversial and is only recommended when it will upstage and change the management of a patient's early-stage disease. ${ }^{26}$

Basic-resource settings. In the absence of capacity to perform staging, patients should be referred to the next level of care. Where that is not possible, immediate symptom control and referral for subsequent care (surgery and/or chemotherapy) should be initiated.

TABLE 5. Summary Diagnosis Recommendations by Setting

\begin{tabular}{|c|c|c|}
\hline \multirow[t]{4}{*}{ Symptoms } & Clinical assessment [S] & $\mathrm{B} / \mathrm{L} / \mathrm{E}$ \\
\hline & $\begin{array}{l}\text { Ultrasound (transabdominal ultrasound, pelvic ultrasound, and TUV), and interpret the results } \\
\text { using the IOTA [S] }\end{array}$ & \\
\hline & CA-125 [M] & \\
\hline & +Contrast-enhanced CT of abdomen and pelvis (with or without thorax) [S] & $\mathrm{L} / \mathrm{E}$ \\
\hline & Histologic confirmation for diagnosis (planning for NACT or not planning for NACT) [M] & $\mathrm{L} / \mathrm{E}$ \\
\hline \multirow{3}{*}{$\begin{array}{l}\text { Staging (suspected early stage vadvanced } \\
\text { stage) }\end{array}$} & Referral for staging surgery [W] & $\mathrm{B} / \mathrm{L}$ \\
\hline & Metastatic workup [W] & $\mathrm{L} / \mathrm{E}$ \\
\hline & Staging surgery $[\mathrm{W}]$ & $\mathrm{E}$ \\
\hline
\end{tabular}

Abbreviations: B, basic; CA-125, cancer antigen 125; CT, computed tomography; E, enhanced; IOTA, International Ovarian Tumor Analysis; L, limited; M, moderate; MRI, magnetic resonance imaging; NACT, neoadjuvant chemotherapy; S, strong; TUV, transvaginal ultrasound; W, weak. 
Limited-resource settings. Diagnostic and surgical staging should proceed as in the Discussion, provided limited settings have appropriate surgical and postoperative expertise and pathology access. Lymph node dissection as part of comprehensive surgical staging for apparent early-stage ovarian cancer includes dissection of pelvic and para-aortic lymph nodes. ${ }^{26}$ Comprehensive surgical assessment for suspected stage I ovarian cancer provides the most accurate information for proper pathology-based staging, as this may affect recommendations for adjuvant chemotherapy options and ultimately survival.

Depending on the histologic grade and subtype, up to $30 \%$ of the patients with apparent early-stage ovarian cancer may be upstaged after pathology results of comprehensive surgical staging. ${ }^{27,28}$

(Sources: SIGN/BGCS, Belgian KCE, BGCS Guidelines, JSGO Guideline)

Enhanced-resource settings. In addition to the recommendations in the basic and limited settings, positron emission tomography-CT is not validated as a diagnostic or staging tool for ovarian cancer.

(Sources: SIGN/BGCS, Belgian KCE, Irish NCEC)

\section{Fertility-sparing surgery and laparoscopic surgery for staging}

Recommendations on fertility-sparing surgery and laparoscopic surgery are in Table 6, Appendix Figures A2 and A8, and the Data Supplement.

\section{Fertility-sparing surgery (Recommendation 2.1.2)}

Discussion. A select subset of patients with stage I and lowgrade ovarian cancer may qualify for fertility-sparing surgery. Where resources allow and in all resource settings, women who wish to preserve fertility options and with apparent early-stage ovarian cancer by imaging should be referred to the highest-level center for presurgery counseling and surgical management by a gynecology oncology surgical specialist. The goal of surgery is to preserve fertility options in addition to performing a comprehensive surgical staging to exclude micrometastasis. In limited-resource settings that lack access to frozen section pathology, a secondary surgery to complete standard procedure may be recommended after confirming final pathology. In the case of stage IB, the patient's uterus can be preserved for future assisted-reproductive options. Women wishing to preserve fertility options should be counseled on the risks of recurrent ovarian cancer on the basis of histology and surgical stage.

Recommendation for fertility-sparing surgery options on the basis of histology:

- Borderline ovarian tumors (clear cell, serous, mucinous, or endometrioid) for stage IA, B, and C

- Mucinous carcinoma for stage IA, B, and C

- Low-grade endometrioid carcinoma for stage IA and B
- Low-grade serous carcinoma for stage IA and B

- Clear cell carcinoma - not recommended for any stage

- High-grade serous or endometrioid tumors-not recommended for any stage

Limited-resource settings and enhanced-resource settings. When young women are affected by early-stage EOC (lowgrade), clinicians can offer fertility-sparing surgery following thorough discussion with the patient about the potential harm of recurrent ovarian cancer.

\section{Laparoscopic surgery for staging (Recommendation 2.1.3)}

Discussion. The preferred approach to surgical staging of suspected ovarian cancer is via a midline vertical incision; data have not yet been provided to validate the safety and equivalence of minimally invasive surgery (MIS) for newly diagnosed EOC care in any resourced setting. The use of laparoscopy for surgical staging in patients with apparent early-stage ovarian cancer is not recommended for basicor limited-resource settings because of the lack of access to expert laparoscopic oncology surgeon(s) and access to necessary equipment for advanced laparoscopy. The safe selection of patients for MIS requires surgical oncology experience beyond laparoscopy surgical techniques. In enhanced settings with capacity for frozen section pathology, MIS may be offered for apparent early-stage ovarian cancer. In such select cases, patients and surgeons must be prepared to convert to a laparotomy procedure if comprehensive surgical staging cannot be completed via MIS.

\section{Surgical debulking for patients diagnosed with stage III and IV ovarian cancer (Recommendation 2.2.1)}

Recommendations on surgical debulking for patients diagnosed with stage III and IV ovarian cancer are in Table 6 and Appendix Figures A2 and A9.

Discussion. The goal of surgical management of stage III and IV ovarian cancer is to perform optimal tumor cytoreduction, which is achieved by resecting all visible tumor to $<1 \mathrm{~cm}$ and ideally to no visible tumor (RO). There is evidence that leaving residual disease $>1 \mathrm{~cm}$ is associated with a reduced chance of cure and negatively affects survival. As the benefit of surgery is diminished with suboptimal cytoreduction, all patients benefit from a multidisciplinary team (MDT) approach to their cancer care. A skilled surgeon should use clinical and radiologic examinations to determine the appropriateness of surgical intervention on the basis of patient and tumor factors with the intent to achieve complete surgical debulking with limited morbidity. Because of the inherent complexity of ovarian cancer surgery, surgeons skilled in oncologic surgery or trained gynecologic oncologists are best positioned to surgically manage patients with stage III or IV ovarian cancer. Decisions to perform surgery for these patients should take into account the health facilities' 
TABLE 6. Summary Treatment Recommendations by Setting

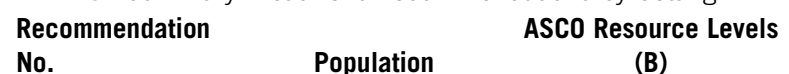

No.

(B)

ASCO Resource Levels (L)

ASCO Resource Levels (E)

Strength of

2.1.1

Low-risk $^{a}$ suspected

Referral to L/E and/or Fertility-sparing surgery if resection if feasible

suspected unilateral

stage I OR refer to

higher-level cancer

center and/or resection

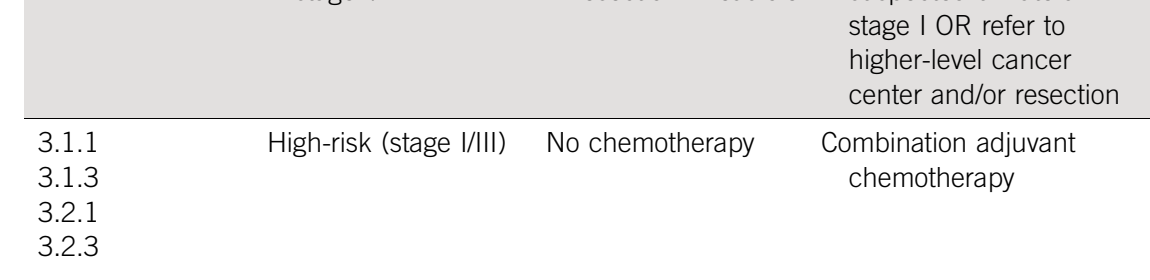

Fertility-sparing surgery if

unilateral stage I. If not, staging

and debulking resection

Recommendation

$\begin{array}{cc}\begin{array}{c}\text { Combination adjuvant } \\ \text { chemotherapy }\end{array} & \begin{array}{c}\text { Moderate/strong } \\ \text { (adjuvant }\end{array} \\ \text { May assess patients with stage III } & \text { chemotherapy) } \\ \text { for appropriate evidence-based } & \text { Weak (targeted } \\ \text { targeted therapy, all patients } & \text { therapy) }\end{array}$

with high-risk features and PS 0-2

\begin{tabular}{|c|c|c|c|c|c|}
\hline $\begin{array}{l}2.2 .2 \\
2.2 .3\end{array}$ & Eligible for $\mathrm{NACT}^{\mathrm{b}}$ & $\begin{array}{l}\text { NACT and interval } \\
\text { debulking is not } \\
\text { recommended in } \\
\text { basic settings }\end{array}$ & $\mathrm{NACT} \leq 4$ cycles & & Weak \\
\hline \multirow[t]{2}{*}{ 2.2.3 A and B } & \multirow{2}{*}{$\begin{array}{l}\text { Metastatic } \\
\text { post-systemic } \\
\text { treatment surgery } \\
\text { (stage IIIC-IV) }\end{array}$} & \multirow{2}{*}{$\begin{array}{l}\text { Interval debulking is } \\
\text { not recommended } \\
\text { in basic settings }\end{array}$} & \multicolumn{2}{|c|}{$\begin{array}{l}\text { (A) If OR or SD to chemotherapy, then interval cytoreductive } \\
\text { debulking surgery }\end{array}$} & \multirow[t]{2}{*}{ Strong } \\
\hline & & & \multicolumn{2}{|c|}{$\begin{array}{l}\text { (B) If PD, alternative chemotherapy regimens, clinical trials, } \\
\text { and/or discontinuation of active cancer therapy and initiation } \\
\text { of end-of-life care }\end{array}$} & \\
\hline $\begin{array}{l}3.1 .1 \\
3.1 .3\end{array}$ & $\begin{array}{l}\text { Received surgery } \\
\quad \text { (adjuvant setting) }^{c}\end{array}$ & $\begin{array}{l}\text { Referral to higher- } \\
\text { level cancer center }\end{array}$ & $\begin{array}{l}\text { If staged and pathologically } \\
\text { confirmed (or alternative } \\
\text { confirmation), then } \\
\text { adjuvant combination } \\
\text { chemotherapy }\end{array}$ & $\begin{array}{l}\text { If staged and pathologically } \\
\text { confirmed (or alternative } \\
\text { confirmation), then adjuvant } \\
\text { combination chemotherapy }\end{array}$ & $\begin{array}{l}\text { Weak } \\
\text { Moderate }\end{array}$ \\
\hline 3.3 .5 & $\begin{array}{l}\text { Received surgery and } \\
\text { prior chemotherapy } \\
\text { (maintenance) } \\
\text { (stage III/IV) }\end{array}$ & NA & NA & $\begin{array}{l}\text { May discuss maintenance } \\
\text { systemic therapies (eg, } \\
\text { antiangiogenic, targeted } \\
\text { therapy). For guidance } \\
\text { regarding the use of PARPi, } \\
\text { please refer to the ASCO } \\
\text { guideline }\end{array}$ & Strong \\
\hline
\end{tabular}

NOTE. Table divided by modality: surgical for primary, surgical for metastatic, etc.

Abbreviations: B, basic; E, enhanced; FIGO, International Federation of Gynecology and Obstetrics; L, limited; NA, not applicable; NACT, neoadjuvant

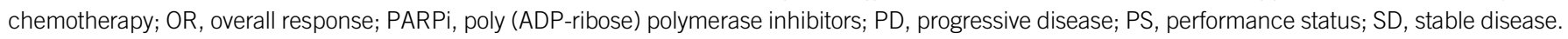

axceptions: ovary appears abnormal and there is evidence of omental and/or peritoneal disease.

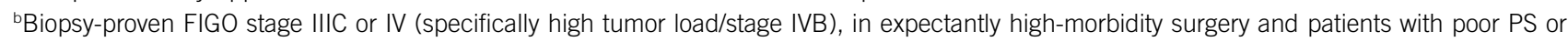
unresectable disease.

'Except low-risk stage I.

capacity to provide safe perioperative care for patients with ovarian cancer because of the underlying risks associated with radical upper abdominal or multiorgan resections, large ascites, pleural effusion, nutritional needs, and patients' preoperative reduced performance status (PS). PS is defined as "A measure of how well a patient is able to perform ordinary tasks and carry out daily activities." 29

The extent of surgery to achieve complete cytoreduction may include bowel resection, upper abdominal exploration, and tumor debulking, or diaphragm resection and is best managed by oncology specialists and at facilities with resources to manage complex postoperative care including pain management. Decisions on bowel resection must consider potential cultural and resource limitations including management of long-term side effects. Routine pelvic and para-aortic lymphadenectomy in surgical management of advanced ovarian cancer (stage III/IV) is not indicated. ${ }^{26}$ Clinically enlarged lymph nodes should be removed as part of debulking procedure. As the surgical decision process for patients' stage III and IV ovarian cancer is complex, this group of patients achieve the best disease outcome when managed by a gynecologic oncologist in the setting of a cancer center and should be referred to the highest-level cancer center for optimal cancer management.

Basic-resource settings. Because of a lack of resources at this level, the Expert Panel recommends that patients 
requiring cytoreductive surgery be referred to higher levels of care.

\begin{abstract}
Limited-resource settings and enhanced-resource settings. Complete cytoreductive surgery for patients with stage III or IV ovarian cancer should be performed by a gynecologic oncologist, general gynecologist, or general surgeon with experience in cancer surgery. Patients should be referred to the highest-level cancer center for optimal surgical management. Access to perioperative supportive services for complex surgical patients may be necessary to optimize surgical outcomes and minimize morbidity.
\end{abstract}

\section{Surgery after neoadjuvant chemotherapy (Recommendation 2.2.2-2.2.3)}

Recommendations on interval cytoreductive surgery after NACT are available in Table 6 and Appendix Figures A3 and $A 9$.

Discussion. Patients with stage III/IV disease with bulky disease may benefit from NACT if a gynecologic oncologist or surgical oncologist reviews the case and deems the patient's disease as unresectable or unlikely to achieve complete cytoreduction. Additional patients appropriate for NACT consideration include those with poor PS or at high surgical risk assessed by a surgical specialist. Research is underway on assessing response to NACT using validated scoring tools and nomograms, although reviewing this literature is outside this guideline's scope..$^{30}$ According to the ASCO and SGO guideline on NACT in stage IIIC and IV, only patients with response to chemotherapy or stable disease following three to four cycles of platinum-based chemotherapy benefit from interval cytoreductive surgery. ${ }^{15}$ The goal of surgery is the same as primary surgery to achieve optimal tumor cytoreduction to $<1 \mathrm{~cm}$, ideally to no visible disease (R0). For patients whose tumor progresses during chemotherapy, interval surgery is not indicated as it offers no added survival benefit. Options for these patients include alternative chemotherapy regimens, clinical trials, and/or discontinuation of active cancer therapy and initiation of end-of-life care. In general, in the setting of progressive disease, there is little role for surgery, and it is not typically advised, unless for palliation (eg, relief of bowel obstruction).

Basic-resource settings. This guideline recommends that patients at this level should be referred to higher levels of care, otherwise patients should be managed with supportive and palliative care interventions.

Limited-resource settings. Treatment response following NACT should be evaluated by an MDT and guided by imaging, tumor marker analysis, and physical examination. Response to NACT may be indicative of a greater likelihood of benefit from interval cytoreductive surgery. Patients with disease response or stable disease benefit from interval cytoreductive surgery to achieve tumor cytoreduction $<1 \mathrm{~cm}$, ideally to no visible disease (R0) where feasible, otherwise achieve tumor cytoreduction to $<1 \mathrm{~cm}$.
Options for patients with progressive disease with NACT are palliative systemic therapies, enrollment in clinical trials, single-agent therapies, or discontinuation of all therapies and pursuit of end-of-life care. There is a laimited role for surgery in patients with poor response to chemotherapy. The decision for additional treatment in patients with progressive disease with NACT should be endorsed by the MDT, weighing the risks and benefits in patients with poor survival outcomes.

Enhanced-resource settings. The same recommendations apply as in limited settings, with added capacity for more aggressive cytoreductive procedures by experienced and specialized surgeons and/or gynecologic oncologists.

\section{OVERARCHING CLINICAL QUESTION C}

What is the optimal adjuvant and/or systemic therapy for stages I-IV EOC?

Recommendations on adjuvant and systemic therapy are in Appendix Figures A3 and A10 and Table 6.

\section{Adjuvant chemotherapy following surgery in patients with} stage I EOC (Recommendations 3.1.1-3.1.5)

Discussion. Stage I ovarian cancer is potentially curable; early and accurate treatment is fundamental to optimizing survival outcomes. Adjuvant chemotherapy may follow any attempt at best possible surgical staging and debulking. Clinicians should use information from surgical staging to guide adjuvant chemotherapy decisions and define disease prognosis. Since patients with previously presumed earlystage ovarian cancer may be upstaged, ${ }^{27,28}$ women who were previously deemed not likely to require adjuvant chemotherapy may qualify for adjuvant therapy given known benefit for OS. ${ }^{31}$ Patients with stage I ovarian cancer who are incompletely staged or completely staged with residual disease experience survival benefit from adjuvant chemotherapy. Adjuvant chemotherapy should not replace additional surgery where feasible.

The OS for stage I EOC is high, although a subset of women is at a higher risk of relapse. Adjuvant chemotherapy does not improve survival for women with stage IA or IB lowgrade (grade 1) endometrioid, serous, or mucinous ovarian carcinoma. This subset of patients is classified as having low-risk early-stage EOC. The risk of relapse is increased with incompletely staged and any grade disease; clear cell, while not normally graded, is considered a high-grade, high-risk histology, and these subsets of patients benefit in improved OS with adjuvant chemotherapy. The international standard-of-care recommendation for adjuvant chemotherapy is a taxane plus platinum doublet on an every-three-weekly schedule (once every 3 weeks) for a total of six cycles; clinicians may use other platinum-based doublets, although there are no data showing noninferiority or superiority to platinum plus taxane doublets. Data exist on the use of three versus six cycles of treatment with nonsignificant difference in survival outcome, although six 
cycles is recommended for stage I high-grade serous histology. Moderately well-differentiated or grade 2 disease can be reclassified as low-grade or high-grade by demonstration of p53 mutation by immunohistochemistry. ${ }^{32-34}$ In the absence of resources to reclassify grade 2 disease on the basis of p53 mutation by immunohistochemistry, adjuvant therapy may be offered to patients with stage I grade 2 EOC. Basic- and limited-resource settings may discuss single-agent platinum therapy for patients with poor PS or in regions with poor access to taxanes and other medications where feasible.

(Sources: SIGN, Belgian KCE, BGSC, OH-CCO, JSGO)

Basic-resource settings and limited-resource settings. Women who have undergone some surgical procedure with diagnosis of possible stage I EOC in basic-resource setting(s) should be referred to a higher-level treatment center for discussion of adjuvant therapy or a restaging procedure. In basic settings, all efforts should be allocated to make this referral. If there are patients for whom referral to a higher level of care is not feasible, close monitoring for recurrence is warranted.

(Source: Consensus)

Limited-resource settings. If extant, an MDT should discuss all patients with stage I disease prior to any intervention. A treatment plan accounting for individual patient characteristics, surgical staging, histology type, and access to chemotherapy should be outlined. Unless contraindicated, patients with stage I eligible for adjuvant chemotherapy should receive it, in the schedule described in the Discussion and in Recommendation 3.1.4 (limited and enhanced settings). Single-agent platinum may be an alternative in select cases.

Enhanced-resource settings. In addition to recommendations under limited settings, women with stage I EOC may have been diagnosed when premenopausal and request fertility preservation. Prior to finalizing surgery and adjuvant chemotherapy treatment plans, women should be referred to a center in an enhanced-resource setting, if available. Consideration of unique management options for patients with stage I ovarian cancer, for example, fertility preservation, the impact of chemotherapy on fertility outcomes, and restaging should be referred to a cancer treatment center with access to highly trained surgeon(s) in enhanced-resource settings.

\section{IP chemotherapy (Recommendation 3.2.2)}

Discussion. IP chemotherapy for stage III ovarian cancer in this guideline is not recommended for basic- or limitedresource settings because of complexity of delivering IP/IV chemotherapy. On the basis of phase III clinical trials, IP/IV chemotherapy confers a survival benefit compared with IV chemotherapy for optimally debulked $(<1 \mathrm{~cm})$ ovarian cancer, with the greatest benefit documented for those with no residual disease (R0) (see the SIGN guideline). ${ }^{12}$ Chemotherapy delivery with paclitaxel plus cisplatin is associated with increased side effects of renal toxicity, neuropathy, fatigue, abdominal discomfort, and infection, frequently leading to early discontinuation of the regimen and change to standard IV chemotherapy. Patients should be counseled about these complications and management options to mitigate side effects, including the need for placement of an IP catheter. IP chemotherapy can be offered in an enhanced setting, for select patients where expertise and supportive services exist to manage toxicities.

Targeted therapy for patients with stage III and IV ovarian cancer (Recommendation 3.2.3 and 3.3.4)

Discussion. This guideline is using the term targeted therapy for bevacizumab and PARPi for the management of EOC. The guideline presumes that these are not available in basic- and limited-resource settings.

A modest PFS benefit and no OS benefits were seen in both randomized phase III trials examining incorporation of the antiangiogenic vascular endothelial growth factor inhibitor, bevacizumab, to platinum-based doublets and continued in maintenance therapy. ${ }^{35,36}$ The subgroups for whom the greatest, albeit still modest, benefit was observed ${ }^{35,36}$ on the basis of data from two large randomized clinical trials, GOG 0218 and ICON-7, were in subsets of patients with ascites or bulky residual disease. ${ }^{35,37,38}$ Bevacizumab can be offered for patients with high risk of disease (stage IV and suboptimal tumor cytoreduction stage III). ${ }^{38}$ Bevacizumab use should be limited to settings with the capacity to closely monitor and manage its known toxicities including hypertension, Gl perforation, arterial or venous thromboembolism, or bleeding. ${ }^{16}$ Use of PARPi is recommended only in more-resourced settings and should follow the ASCO guidelines for use of PARPi in ovarian cancer. ${ }^{21}$

Basic-resource settings. Targeted therapy is not recommended in basic settings.

Limited-resource settings. Targeted therapy is not recommended in limited-resource settings because of issues of access and toxicity, including financial toxicity.

Enhanced-resource settings. For enhanced settings, bevacizumab is an option, where feasible for appropriately selected stage III/IV patients. Cost and safety concerns with the management of toxicities may limit applicability.

\section{Maintenance systemic therapy (Recommendation 3.3.5)}

Recommendations on maintenance systemic therapy for patients with stage III/IV ovarian cancer after adjuvant chemotherapy are available in Appendix Figure A4 and Table 6.

Discussion. The benefit of maintenance chemotherapy with antiangiogenic vascular endothelial growth factor inhibitor bevacizumab in all subgroups of patients is still under debate in the absence of strong data; see the Discussion under Recommendation 3.2.3. ${ }^{38}$ In patients with 
stage II to IV low-grade serous histology, maintenance hormonal therapy can be discussed; the data are limited, and there is no clearly documented OS benefit at the time of this writing. ${ }^{39}$ As new information continues to evolve, future updates of this and other ASCO guidelines will discuss other new agents including PARPi, following enough evidence of efficacy. The major limitation to maintenance therapy such as PARPi in resource-constrained settings is lack of access to biomarker testing, including identification of patients with homologous recombination repair deficiency (HRD) and, more specifically, patients with tumors exhibiting BRCA mutation. Where biomarker testing results indicate treatment, continuous access to medication and cost-effectiveness analysis specific to resource settings must be ensured.

Limited-resource settings. In limited-resource settings, maintenance therapy with antiangiogenic agents is not recommended because of cost limitations and clinician experience in toxicity management.

Enhanced-resource settings. In enhanced-resource settings, clinicians may discuss maintenance therapy with antiangiogenic agents with select patients (those with stage III/IV disease) and potentially PARPi (for latter, see ASCO PARPi guideline, JCO, 2020). ${ }^{21}$ Institutions that use maintenance treatment with antiangiogenic and/or PARPi agents need capacity for evaluation and management of side effects.

\section{OVERARCHING CLINICAL QUESTION D}

What is the optimal therapy for women with recurrent EOC? (see Table 7 and Appendix Figures A5, A6, and A10)

\section{Discussion}

Despite the initial success with first-line surgery and chemotherapy for ovarian cancer, most patients will develop recurrent disease. ${ }^{12}$ The risk of recurrence is highest with advanced and high-grade or clear cell disease. Recurrent ovarian cancer is stratified into platinum-sensitive or platinum-resistant on the basis of the length of time to relapse ( $\geq 6$ months $v<6$ months, respectively) from the end of treatment with first-line platinum-based chemotherapy. Chemotherapy is the primary intervention for recurrent disease; where chemotherapy is not feasible for any reason, palliative care, if not already invoked, should be initiated.

(Source: BCGS, OH-CCO, SIGN)

\section{Surgery for recurrent EOC (Recommendation 4.0)}

Recommendations on surgery for patients with recurrence are in Table 7.

TABLE 7. Summary Treatment of Recurrent EOC Recommendations by Setting

\begin{tabular}{|c|c|c|c|c|c|}
\hline $\begin{array}{l}\text { Recommendation } \\
\text { No. }\end{array}$ & Population & $\begin{array}{l}\text { ASCO Resource } \\
\text { Levels (B) }\end{array}$ & ASCO Resource Levels (L) & ASCO Resource Levels (E) & $\begin{array}{c}\text { Strength of } \\
\text { Recommendation }\end{array}$ \\
\hline 4.0 & $\begin{array}{l}\text { Recurrent small-volume } \\
\text { platinum-sensitive disease } \\
\text { EOC }\end{array}$ & $\begin{array}{l}\text { Because of health } \\
\text { system gaps, not } \\
\text { feasible in the } \\
\text { basic setting }\end{array}$ & $\begin{array}{l}\text { Refer to higher-level } \\
\text { cancer center for } \\
\text { surgical consideration }\end{array}$ & $\begin{array}{l}\text { Perform complete } \\
\text { secondary cytoreductive } \\
\text { debulking surgery }\end{array}$ & Moderate: L/E \\
\hline $4.1 / 4.4$ & $\begin{array}{l}\text { Recurrent EOC who have } \\
\text { received prior systemic } \\
\text { treatment }\end{array}$ & $\begin{array}{l}\text { Best supportive } \\
\text { care }\end{array}$ & Early palliative care & Early palliative care & Strong \\
\hline $4.1 / 4.2$ & $\begin{array}{l}\text { Recurrent platinum-sensitive, } \\
\text { platinum-resistant, platinum- } \\
\text { refractory EOC-who have } \\
\text { received prior systemic } \\
\text { treatment, PS 0-2 }\end{array}$ & N/A & $\begin{array}{l}\text { Treatment with second- } \\
\text { line chemotherapy or } \\
\text { refer to higher-level } \\
\text { cancer center }\end{array}$ & $\begin{array}{l}\text { Treatment with second-line } \\
\text { chemotherapy }\end{array}$ & $\begin{array}{l}\text { Moderate: L } \\
\text { Strong: E }\end{array}$ \\
\hline \multirow[t]{3}{*}{4.2} & Platinum-sensitive EOC & $N / A$ & $\begin{array}{l}\text { Platinum-sensitive: } \\
\text { combination } \\
\text { chemotherapy with } \\
\text { carboplatin }\end{array}$ & $\begin{array}{l}\text { Platinum-sensitive: } \\
\text { combination } \\
\text { chemotherapy with } \\
\text { carboplatin with or without } \\
\text { bevacizumab }\end{array}$ & Strong: L/E \\
\hline & Platinum-resistant EOC & $N / A$ & $\begin{array}{l}\text { Single-agent nonplatinum } \\
\text { chemotherapy or best } \\
\text { supportive care }\end{array}$ & $\begin{array}{l}\text { Single-agent nonplatinum } \\
\text { chemotherapy with or } \\
\text { without bevacizumab or } \\
\text { best supportive care }\end{array}$ & $\begin{array}{l}\text { Resistant } \\
\text { Strong: L/E }\end{array}$ \\
\hline & Platinum-refractory EOC & $N / A$ & & $\begin{array}{l}\text { Single-agent nonplatinum } \\
\text { chemotherapy with or } \\
\text { without biologic agent } \\
\text { (bevacizumab) }\end{array}$ & $\begin{array}{l}\text { Refractory } \\
\text { Moderate: E }\end{array}$ \\
\hline
\end{tabular}

Abbreviations: B, basic; E, enhanced; EOC, epithelial ovarian cancer; L, limited; N/A, not available; PS, performance status. 


\section{Discussion}

Limitations in access to advanced therapies in resourceconstrained settings support the discussion of secondary cytoreductive surgery in select patients with recurrent ovarian cancer. General agreement is that this intervention should only be considered for women with platinumsensitive, delayed tumor recurrence, limited disease, good underlying performance and end organ status, and access to optimal surgical and postoperative support. Prognostic factors associated with best surgical outcomes are isolated site(s) of tumor recurrence and limited ascites $(<500 \mathrm{~mL})$.

Secondary cytoreduction can be considered for appropriately selected patients. The research describing the potential benefit of secondary debulking surgery is ongoing; however, given the level of expertise with this procedure and the need of an MDT to proceed with this type of surgery, it should not be performed in a resource setting other than maximal.

\section{Basic-resource settings}

Patients with recurrent disease should be referred to a higher-level care center wherever possible and to palliative care if not already started. Although there is a limited role for secondary cytoreductive surgery in recurrent ovarian cancers, this may be the only therapeutic opportunity to ameliorate symptoms for women in basic-resource settings.

\section{Limited-resource settings}

In settings where systemic therapy is not readily available and a skilled general surgeon or gynecologist trained in appropriate skills in oncologic surgery is available, evaluation and surgical management for recurrent ovarian cancer can be considered as one approach to disease management. All patients should be evaluated by an MDT, if available, to avoid unnecessary and risky surgical intervention.

(Source: JSGO Guideline, BCGS, SIGN)

\section{Enhanced-resource settings}

The proper care for women with recurrent ovarian cancer, with the exception of very limited resource situations, is systemic therapy. Secondary cytoreductive surgery can be discussed for highly selected platinum-sensitive patients; survival benefit is limited to patients for whom clinicians can achieve complete cytoreductive surgery. For all other patients with ovarian cancer, surgery should be withheld, except for symptom management such as for limited bowel obstruction readily overcome with diversion.

(Source: BCGS)

\section{Systemic and palliative treatment for recurrent EOC (Recommendations 4.1-4.4)}

Recommendations on systemic treatment and palliative care for patients with recurrent ovarian cancer are in Table 7.

Discussion. Systemic therapy is the cornerstone of managing patients with recurrent disease. Approaches to recurrent disease will vary with resource availability to multiple chemotherapy agents, location and severity of recurrence, and prior treatment exposures. Recurrent disease is not amenable to cure but patients with platinum sensitivity have improved PFS and OS when re-treated with platinum-based single-agent or doublet therapy. Few if any opportunities may be available to women with recurrent disease in basic-resourced areas and palliative care may be the only option. Surgery is not recommended except in select patients who had complete resection at up-front surgery, long recurrence-free survival, and limited disease at re-presentation. ${ }^{26}$ Decisions for surgery in recurrent EOC must take into account patient risk factors, access to qualified surgical capacity, system-based resources, and access to second-line chemotherapy agents. Systemic chemotherapy and targeted therapy recommendations depend upon resource support and platinum-free interval (Table 8).

Cost, access, and safety issues with targeted therapies such as bevacizumab are of concern in limited and enhanced settings, although there may be benefits in management of ascites. Bowel perforation, thromboembolism, fatigue, and hypertension are grade 3 and 4 toxicities occurring in patients receiving bevacizumab and are of grave concern, especially in weaker health systems. Clinical trials and newer targeted agents may be available in some limited and enhanced settings. Patients should be referred to higherresourced settings for these and other opportunities when eligible and feasible.

Patients in the platinum-resistant group do not derive benefit from platinum rechallenge. The current standard of

TABLE 8. Risk-Stratified Groups

\section{Platinum-Sensitive}

\section{Platinum-Resistant}

Failure in $<6$ months following completion of platinum-based chemotherapy

\section{Platinum-Refractory}

Disease progression while receiving first-line platinum-based chemotherapy

Patients with no prior platinum-based therapy are also in this group

NOTE. Platinum-sensitive if the platinum-free interval is 6 months or more; some guidelines have a partially sensitive group if the platinum-free interval is between 6 and 12 months. There are no approvals internationally that apply this definition (Ontario Health—Cancer Care Ontario). ${ }^{16}$ 
TABLE 9. Selected Limitations and Future Directions From Adapted Guidelines

\begin{tabular}{ll}
\hline Role of tumor histology & JSGO \\
\hline MRI for staging & Irish NCEC \\
\hline Maintenance, especially with novel agents & Multiple \\
\hline $\begin{array}{l}\text { Diagnostic accuracy of secondary tests, including } \\
\text { reproducibility of tests involving ultrasound }\end{array}$ & $\begin{array}{c}\text { Tests in secondary care to identify people at high risk of ovarian cancer. Diagnostics guidance } \\
\text { [DG31]. Published date: November 15, 2017. URL: https://www.nice.org.uk/guidance/ } \\
\text { dg31/chapter/6-Recommendations-for-further-research. Page last updated: NR. }\end{array}$ \\
& Accessed: June 22, 2020 \\
\hline Analyses of existing data sets of secondary tests & Tests in secondary care to identify people at high risk of ovarian cancer. Diagnostics guidance \\
& [DG31]. Published date: November 15, 2017. URL: https://www.nice.org.uk/guidance/ \\
& dg31/chapter/6-Recommendations-for-further-research. Page last updated: NR. \\
\hline Validating Chemotherapy Response Score and & Accessed: June 22, 2020 \\
consequent risk stratification & ASCO/SGO \\
\hline Use of (weekly) paclitaxel for NACT & ASCO/SGO \\
\hline Novel agents in NACT & ASCO/SGO \\
\hline Minimally invasive techniques & JSGO \\
\hline Role of IP chemotherapy & Multiple
\end{tabular}

Abbreviations: IP, intraperitoneal; JSGO, Japan Society of Gynecologic Oncology; MRI, magnetic resonance imaging; NACT, neoadjuvant chemotherapy; NCEC, National Clinical Effectiveness Committee; NR, not reported; SGO, Society of Gynecologic Oncology.

care outside of a clinical trial is single-agent non-platinumbased chemotherapy with or without bevacizumab in maximal settings. An exception is the use of hormonal therapy for recurrent low-grade serous (and low-grade endometrioid) ovarian cancer, where the pathologic diagnosis and grade are confirmed. The platinum-refractory group has a poor prognosis with short disease-free intervals and should be managed with palliative intent (Table 7).

(Sources: BCGS, OH-CCO, JSGO, SIGN)

Basic-resource settings. Palliative care involvement, if not already initiated, should be the primary focus alone or along with referral to higher-resourced settings.

Limited-resource settings. Access to an MDT, systemic therapies, and oncology expertise is likely inconsistent in most limited-resource settings. Patients should be managed on the basis of platinum-free intervals, ability to tolerate additional chemotherapy, treatment and palliative support, and access to nonplatinum agents. Toxicity profiles of recommended therapies should be discussed with the patient including available options for management of toxicities.

Enhanced-resource settings. Recommendations for recurrent EOC outlined for limited-resource settings are applicable in the enhanced settings. When an MDT endorses targeted therapies, including antiangiogenic agents or PARPi (see ASCO guideline), a clinician may discuss these options with patients. The strength of the health system determines the feasibility of administering targeted agents. Outcomes should be balanced with QoL including financial toxicity. ASCO has developed new guidelines for the use of targeted therapies including PARP ${ }^{21}$ in the management of ovarian cancer in maximal settings and these can be discussed in enhanced-resource settings where applicable.

\section{SPECIAL COMMENTARY}

\section{Pathology}

Pathology is an important part of diagnosing the type of EOC and guiding management of women with this disease. There is variable availability and financing for pathology services around the world. In some regions, clinicians may even have to make diagnoses without pathology. ASCO resource-stratified guidelines use the capacity framework in Table 2 to guide pathology recommendations. As resource-constrained regions develop pathology services, the Expert Panel would like to make some suggestions specific to ovarian cancer.

The clinical presentation and imaging findings of both benign (eg, peritoneal tuberculosis) and other malignancies (eg, Gl cancer) may be similar or mimic those of ovarian cancer. Consequently, a histopathologic diagnosis should be undertaken prior to definitive treatment. Pathologic diagnosis may be rendered on a peritoneal or omental biopsy, particularly in patients for whom there is the potential for neoadjuvant intervention, or on resection specimens following laparotomy or laparoscopy. Usually, routine histologic processing of formalin-fixed tissue is sufficient for pathologic diagnosis. Immunohistochemical studies may provide additional confirmatory evidence, but are often not critical to diagnosis. Alternatively, a cytopathologic diagnosis may be enough if this specialized service 
is available. In some limited and enhanced settings, ascitic fluid can be sent to pathology for cell block in major cities. Where laboratories are of variable quality, cytology alone can be problematic. In some cases, immunohistochemical tests can be sent to a central laboratory to confirm diagnosis, especially if a sample is mucinous.

ASCO believes that cancer clinical trials are vital to inform medical decisions and improve cancer care and that all patients should have the opportunity to participate. The expansion of oncology clinical trials in limited and enhanced settings is a global oncology priority.

\section{COST IMPLICATIONS}

An ASCO literature search focusing on high-quality systematic reviews of published cost-effectiveness analyses in low-resource settings was conducted, and none were found.

\section{LIMITATIONS OF THE RESEARCH AND FUTURE DIRECTIONS}

There were limitations on the evidence to inform some of the recommendations because of many recognizable factors such as prioritization of patient care and limited funding and infrastructure for research.

Limitations include insufficient research conducted in resource-constrained settings, lack of conclusive research on primary/prevention screening, lack of published data on ovarian cancer genetic risk evaluation, and management adapted to resource-constrained settings. Expert recommendations for resource-constrained settings should account for differential access to chemotherapy across basicand limited-resource settings. A shortage in human resources of trained gynecologic oncologists has led to taskshifting with variation in skill set among general practitioners, obstetricians/gynecologists, general surgeons, and oncologists able to manage patients with ovarian cancer.

There is a significant need to further ovarian cancer research in resource-constrained settings, considering issues of surgery and chemotherapy access, treatment effectiveness, and cost-effectiveness. The paucity of ovarian cancer genetic research in limited-resource settings needs further investigation, which can be achieved through collaborative research. The use of targeted therapy in adjuvant, maintenance, and recurrent ovarian cancer is actively under investigation, and further guidelines will include updates. Further limitations are listed in Table 9.

\section{EXTERNAL REVIEW AND OPEN COMMENT}

The draft recommendations were released to the public for open comment from June 29 through July 13, 2020. Response categories of "Agree as written," "Agree with suggested modifications," and "Disagree. See comments" were captured for every proposed recommendation with 10 written comments received. A total of $90 \%$ of the 10 respondents either agreed or agreed with slight modifications to the recommendations and $10 \%$ of the respondents disagreed. Expert Panel members reviewed comments from all sources and determined whether to maintain original draft recommendations, revise with minor language changes, or consider major recommendation revisions. All changes were incorporated prior to CPGC review and approval.

The draft was submitted to six external reviewers with content expertise; two completed the reviews. It was rated as high quality, and it was agreed it would be useful in practice. Review comments were reviewed by the Expert Panel and integrated into the final manuscript before approval by the CPGC.

\section{GUIDELINE IMPLEMENTATION}

ASCO guidelines are developed for implementation across health settings. Barriers to implementation include the need to increase awareness of the guideline recommendations among frontline practitioners and patients with ovarian cancer and to provide adequate services in the face of limited resources. The guideline Bottom Line was designed to facilitate implementation of recommendations. This guideline will be distributed widely, including through many forms of ASCO communications and the ASCO website.

\section{ADDITIONAL RESOURCES}

Additional information including a supplement, evidence tables, and clinical tools and resources can be found at www.asco.org/resource-stratified-guidelines. Patient information is available there and at www.cancer.net.

\section{RELATED ASCO GUIDELINES}

\section{Resource-Stratified Guidelines}

- Palliative Care in the Global Setting40 (http://ascopubs.org/doi/10.1200/JG0.18.00026)

\section{Non-Resource-Stratified Guidelines}

- Neoadjuvant Chemotherapy for Ovarian Cancer $^{14,15}$ (https://ascopubs.org/doi/full/10.1200/ JC0.2016.68.6907)

- Germline and Somatic Tumor Testing in Epithelial Ovarian Cancer $^{20}$ (https://ascopubs.org/doi/10. 1200/JC0.19.02960)

- Integration of Palliative Care into Standard Oncology Practice ${ }^{41} \quad$ (http://ascopubs.org/doi/10. 1200/JC0.2016.70.1474)

- PARP Inhibitors in the Management of Ovarian Cancer ${ }^{21}$ (http://ascopubs.org/doi/10.1200/JC0.20.01924)

- Patient-Clinician Communication ${ }^{42} \quad$ (http:// ascopubs.org/doi/10.1200/JC0.2017.75.2311) 


\section{AFFILIATIONS}

${ }^{1}$ Korlebu Teaching Hospital, Accra, Ghana

${ }^{2}$ Hospital Angeles Del Pedregal, Mexico City, Mexico

${ }^{3}$ American Society of Clinical Oncology, Alexandria, VA

${ }^{4}$ Hameed Latif Hospital, Lahore, Pakistan

${ }^{5}$ Stony Brook University Hospital, Stony Brook, NY

${ }^{6}$ Sociedad Lucha Contra Cancer Ecuador, Machala, Ecuador

${ }^{7}$ Nuvance Health System, Danbury, CT

${ }^{8}$ National Cancer Institute, Bethesda, MD

${ }^{9}$ LifeLabs, Ontario, Canada

${ }^{10}$ Massachusetts General Hospital, Boston, MA

${ }^{11}$ Saitama Medical University International Medical Center, Saitama, Japan

${ }^{12}$ Instituto Nacional de Cancerología, La Paz, Bolivia

${ }^{13}$ Komfo Anokye Teaching Hospital, Kumasi, Ghana

${ }^{14}$ Chittaranjan National Cancer Institute, Kolkata, India

${ }^{15}$ Northern Gynaecological Oncology Centre, Gateshead, Newcastle, United Kingdom

${ }^{16}$ NAMS, Bir Hospital, Kathmandu, Nepal

${ }^{17}$ Mallow Flower Foundation, Dunaharaszti, Hungary

${ }^{18}$ Ramathibodi Hospital, Mahidol University, Bangkok, Thailand

${ }^{19}$ University of Minnesota Medical School, Minneapolis, MN

${ }^{20}$ St Paul's Hospital Millennium Medical School, Addis Ababa, Ethiopia

\section{CORRESPONDING AUTHOR}

American Society of Clinical Oncology, 2318 Mill Rd, Suite 800, Alexandria, VA 22314; email: guidelines@asco.org.

\section{EDITOR'S NOTE}

This ASCO Guideline provides recommendations, with comprehensive review and analyses of the relevant literature for each recommendation. Additional information, including a supplement with additional evidence tables, slide sets, clinical tools and resources, and links to patient information at www.cancer.net, is available at www.asco.org/resourcestratified-guidelines.

\section{EQUAL CONTRIBUTION}

J.R.V.C. and V.D.V. were cochairs and are cofirst authors recognizing equal contributions. R.G.G. was the third member of the writing subcommittee, along with the cochairs.

\section{AUTHOR CONTRIBUTIONS}

Conception and design: All authors

Administrative support: Sarah Temin

Collection and assembly of data: All authors

Data analysis and interpretation: Verna D. Vanderpuye, Jean Rene V.

Clemenceau, Sarah Temin, Elise C. Kohn, Asima Mukhopadhyay, Rahel

G. Ghebre

Manuscript writing: All authors

Final approval of manuscript: All authors

Accountable for all aspects of the work: All authors

\section{AUTHORS' DISCLOSURES OF POTENTIAL CONFLICTS OF INTEREST}

The following represents disclosure information provided by the authors of this manuscript. All relationships are considered compensated unless otherwise noted. Relationships are self-held unless noted. I = Immediate Family Member, Inst = My Institution. Relationships may not relate to the subject matter of this manuscript. For more information about ASCO's conflict of interest policy, please refer to www.asco.org/rwc or ascopubs. org/go/authors/author-center.

Open Payments is a public database containing information reported by companies about payments made to US-licensed physicians (Open Payments).

Terence J. Colgan

Consulting or Advisory Role: LifeLabs

Research Funding: MT Pharma

Expert Testimony: J and J

Other Relationship: College of American Pathologists

Open Payments Link: https://openpaymentsdata.cms.gov/physician/ xxxxxxx/summary

Keiichi Fujiwara

Honoraria: Kyowa Hakko Kirin, Zeria Pharmaceutical, Nippon Kayaku, Chugai Pharma, Eisai, Taiho Pharmaceutical, Daiichi Sankyo, Ono Pharmaceutical, Takeda

Consulting or Advisory Role: AstraZeneca, MSD, Taiho Pharmaceutical, Eisai, Takeda, Genmab, AbbVie, Pfizer

Research Funding: Eisai, Kaken Pharmaceutical, Chugai Pharma, Shionogi, Immunogen, Oncotherapeutics, AstraZeneca, Lilly, Zeria Pharmaceutical, Ono Pharmaceutical, MSD, Genmab, Regeneron Travel, Accommodations, Expenses: Pfizer, AbbVie, MSD

\section{Asima Mukhopadhyay}

Research Funding: Meda

Patents, Royalties, Other Intellectual Property: Royalty payment from Clovis Oncology for contribution toward development of rucaparib as a member of the Newcastle University drug development team

Icó Tóth

Honoraria: MSD, SYNLAB

Travel, Accommodations, Expenses: Roche Magyarország Kft, Roche Diagnostic Kft Hungary

Other Relationship: AstraZeneca, Roche Magyarország Kft, MSD, Roche, Roche Diagnostic Kft Hungary, SYNLAB Kft Hungary, Roche Pharma AG, GlaxoSmithKline

Rahel G. Ghebre

Stock and Other Ownership Interests: Gilead Sciences

No other potential conflicts of interest were reported.

\section{ACKNOWLEDGMENT}

The Expert Panel wishes to thank external reviewers Dr Nathalie McKenzie, Dr Rodney Rocconi, and Dr Hannah Simonds; CPGC reviewers Dr Raetasha Dabney and Dr Jamie Lee Lesnock; and the Clinical Practice Guidelines Committee for their thoughtful reviews and insightful comments on this guideline adaptation. The Expert Panel also thanks the Consensus Panel members for their review of the guideline recommendations. 


\section{REFERENCES}

1. United Nations Development Programme: Human Development Reports: Human Development Index http://hdr.undp.org/en/content/human-developmentindex-hdi

2. Bray F, Ferlay J, Soerjomataram I, et al: Global cancer statistics 2018: GLOBOCAN estimates of incidence and mortality worldwide for 36 cancers in 185 countries. CA Cancer J Clin 68:394-424, 2018

3. Chornokur G, Amankwah EK, Schildkraut JM, et al: Global ovarian cancer health disparities. Gynecol Oncol 129:258-264, 2013

4. Malvezzi M, Carioli G, Rodriguez T, et al: Global trends and predictions in ovarian cancer mortality. Ann Oncol 27:2017-2025, 2016

5. Chuang L, Berek J, Randall T, et al: Collaborations in gynecologic oncology education and research in low- and middle- income countries: Current status, barriers and opportunities. Gynecol Oncol Rep 25:65-69, 2018

6. Al-Sukhun S, Temin S, Chavez-MacGregor M, et al: ASCO resource-stratified guidelines: Methods and opportunities. J Glob Oncol 4:1-8, 2018

7. Anderson BO, Shyyan R, Eniu A, et al: Breast cancer in limited-resource countries: An overview of the Breast Health Global Initiative 2005 guidelines. Breast J 12:S3-S15, 2006 (suppl 1)

8. Horton S, Gauvreau CL: Cancer in low- and middle-income countries: An economic overview, in Gelband H, Jha P, Sankaranarayanan R, et al (eds): Cancer: Disease Control Priorities, Volume 3 (ed 3). Washington, DC, International Bank for Reconstruction and Development/World Bank, 2015

9. The ADAPTE Collaboration: The ADAPTE process: resource toolkit for guideline adpatation. Version 2.0: Guideline International Network; 2009. http://www.g-in.net/document-store/working-groups-documents/adaptation/adapte-resource-toolkit-guideline-adaptation-2-0.pdf. Objective of the ADAPTE process. http:// www.adapte.org/

10. Shiffman RN, Michel G, Rosenfeld RM, et al: Building better guidelines with BRIDGE-Wiz: Development and evaluation of a software assistant to promote clarity, transparency, and implementability. J Am Med Inform Assoc 19:94-101, 2012

11. Guidelines International Network: International Guidelines Library. https://guidelines.ebmportal.com/ G-I-N International Guideline Library. G-I-N.net

12. Scottish Intercollegiate Guidelines Network (SIGN): Management of Epithelial Ovarian Cancer. Edinburgh, Scotland, Scottish Intercollegiate Guidelines Network, 2013. (SIGN publication no. 135). [November 2013]. http://www.sign.ac.uk, Edinburgh, 2018

13. Vergote I, Vlayen J, Heus P: Ovarian Cancer: Diagnosis, Treatment and Follow-up. Good Clinical Practice (GCP) Brussels: Belgian Health Care Knowledge Centre (KCE), 2016. KCE Reports 268. D/2016/10.273/49

14. Wright AA, Bohlke K, Armstrong DK, et al: Neoadjuvant chemotherapy for newly diagnosed, advanced ovarian cancer: Society of Gynecologic Oncology and American Society of Clinical Oncology Clinical Practice Guideline. Gynecol Oncol 143:3-15, 2016

15. Wright AA, Bohlke K, Armstrong DK, et al: Neoadjuvant chemotherapy for newly diagnosed, advanced ovarian cancer: Society of Gynecologic Oncology and American Society of Clinical Oncology Clinical Practice Guideline. J Clin Oncol 34:3460-3473, 2016

16. Francis J, Coakley N, Elit L, et al: Systemic Therapy for Recurrent Ovarian Cancer. Toronto, ON, Cancer Care Ontario, 2017. Program in Evidence-Based Care Guideline No.: 4-3v4

17. Komiyama S, Katabuchi H, Mikami M, et al: Japan Society of Gynecologic Oncology guidelines 2015 for the treatment of ovarian cancer including primary peritoneal cancer and fallopian tube cancer. Int J Clin Oncol 21:435-446, 2016

18. Fotopoulou C, Hall M, Cruickshank D, et al: British Gynaecological Cancer Society (BGCS) epithelial ovarian/fallopian tube/primary peritoneal cancer guidelines: Recommendations for practice. Eur J Obstet Gynecol Reprod Biol 213:123-139, 2017

19. Sláinte AR, The Department of Health: Diagnosis and staging of patients with ovarian cancer (NCEC National Clinical Guideline No. 20). 2019. https://health. gov.ie/national-patient-safety-office/ncec/nationalclinical-guidelines/

20. Konstantinopoulos PA, Norquist B, Lacchetti C, et al: Germline and somatic tumor testing in epithelial ovarian cancer: ASCO guideline. J Clin Oncol 38:1222-1245, 2020

21. Tew WP, Lacchetti C, Ellis A, et al: PARP inhibitors in the management of ovarian cancer: ASCO guideline. J Clin Oncol 38:3468-3493, 2020

22. Loblaw DA, Prestrud AA, Somerfield MR, et al: American Society of Clinical Oncology Clinical Practice Guidelines: Formal systematic review-based consensus methodology. J Clin Oncol 30:3136-3140, 2012

23. Chiorean EG, Nandakumar G, Fadelu T, et al: Treatment of patients with late-stage colorectal cancer: ASCO resource-stratified guideline. JCO Glob Oncol 6:414-438, 2020

24. Ebell MH, Culp MB, Radke TJ: A systematic review of symptoms for the diagnosis of ovarian cancer. Am J Prev Med 50:384-394, 2016

25. Robson ME, Bradbury AR, Arun B, et al: American Society of Clinical Oncology policy statement update: Genetic and genomic testing for cancer susceptibility. J Clin Oncol 33:3660-3667, 2015

26. Harter P, Sehouli J, Lorusso D, et al: A randomized trial of lymphadenectomy in patients with advanced ovarian neoplasms. N Engl J Med 380:822-832, 2019

27. Young RC, Decker DG, Wharton JT, et al: Staging laparotomy in early ovarian cancer. JAMA 250:3072-3076, 1983

28. Schuueler JA, Trimbos JB, Hermans J, et al: The yield of surgical staging in presumed early stage ovarian cancer. Int J Gynecol Cancer 8:95-102, 1998

29. National Cancer Institute: Comprehensive Cancer Information. www.cancer.gov

30. Liang WF, Li H, Wu JY, et al: Identification of ovarian cancer patients most likely to achieve chemotherapy response score 3 following neoadjuvant chemotherapy: Development of a predictive nomogram. Front Oncol 10:560888, 2020

31. Garcia-Soto AE, Boren T, Wingo SN, et al: Is comprehensive surgical staging needed for thorough evaluation of early-stage ovarian carcinoma? Am J Obstet Gynecol 206:242.e1-242.e5, 2012

32. Altman AD, Nelson GS, Ghatage P, et al: The diagnostic utility of TP53 and CDKN2A to distinguish ovarian high-grade serous carcinoma from low-grade serous ovarian tumors. Mod Pathol 26:1255-1263, 2013

33. Vang R, Levine DA, Soslow RA, et al: Molecular alterations of TP53 are a defining feature of ovarian high-grade serous carcinoma: A rereview of cases lacking TP53 mutations in the cancer genome atlas ovarian study. Int J Gynecol Pathol 35:48-55, 2016

34. Levesque MA, Katsaros D, Yu H, et al: Mutant p53 protein overexpression is associated with poor outcome in patients with well or moderately differentiated ovarian carcinoma. Cancer 75:1327-1338, 1995

35. Oza AM, Cook AD, Pfisterer J, et al: Standard chemotherapy with or without bevacizumab for women with newly diagnosed ovarian cancer (ICON7): Overall survival results of a phase 3 randomised trial. Lancet Oncol 16:928-936, 2015

36. Tewari KS, Burger RA, Enserro D, et al: Final overall survival of a randomized trial of bevacizumab for primary treatment of ovarian cancer. J Clin Oncol 37:2317-2328, 2019

37. Burger RA, Brady MF, Bookman MA, et al: Incorporation of bevacizumab in the primary treatment of ovarian cancer. N Engl J Med 365:2473-2483, 2011

38. Perren TJ, Swart AM, Pfisterer J, et al: A phase 3 trial of bevacizumab in ovarian cancer. N Engl J Med 365:2484-2496, 2011 
39. Gershenson DM, Bodurka DC, Coleman RL, et al: Hormonal maintenance therapy for women with low-grade serous cancer of the ovary or peritoneum. J Clin Oncol 35:1103-1111, 2017

40. Osman H, Shrestha S, Temin S, et al: Palliative care in the global setting: ASCO resource-stratified practice guideline. J Glob Oncol 4:1-24, 2018

41. Ferrell BR, Temel JS, Temin S, et al: Integration of palliative care into standard oncology care: American Society of Clinical Oncology Clinical Practice Guideline update. J Clin Oncol 35:96-112, 2017

42. Gilligan T, Coyle N, Frankel RM, et al: Patient-clinician communication: American Society of Clinical Oncology consensus guideline. J Clin Oncol 35:3618-3632, 2017 


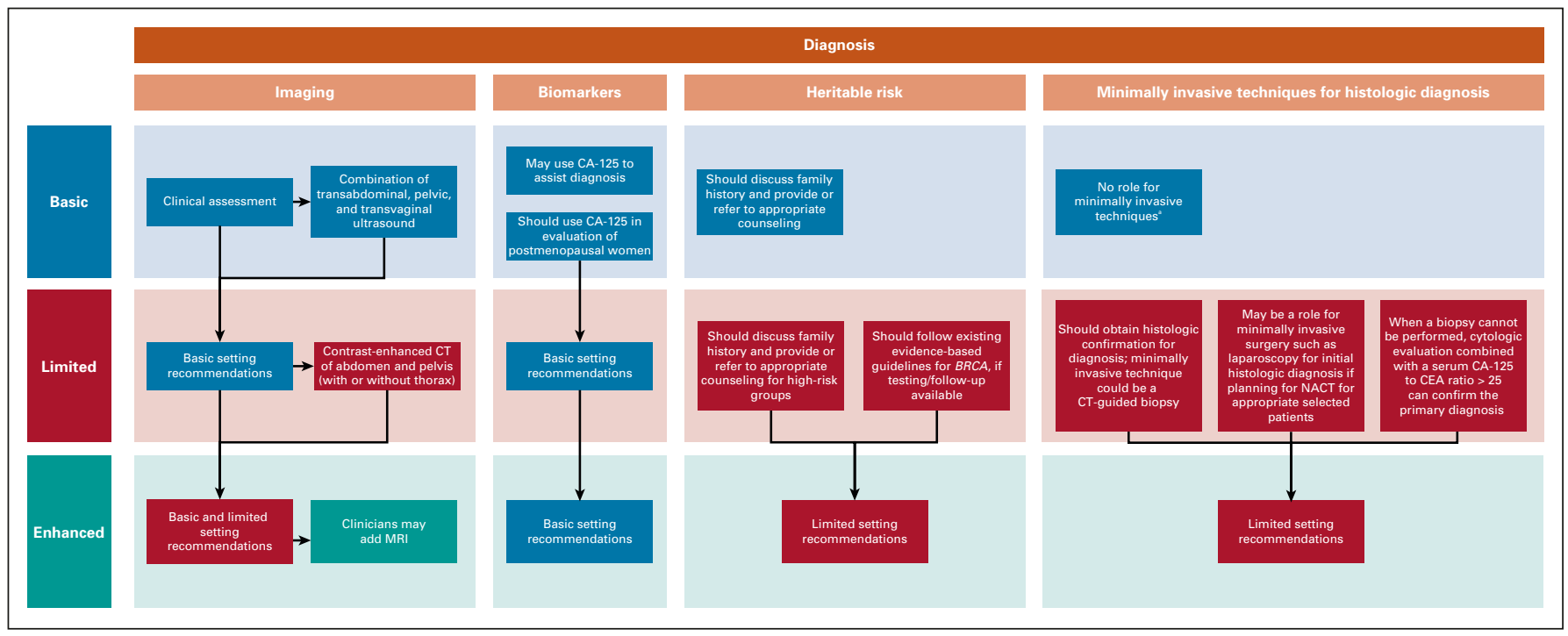

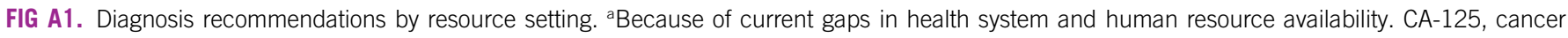
antigen 125; CEA, carcinoembryonic antigen; CT, computed tomography; MRI, magnetic resonance imaging; NACT, neoadjuvant chemotherapy. 


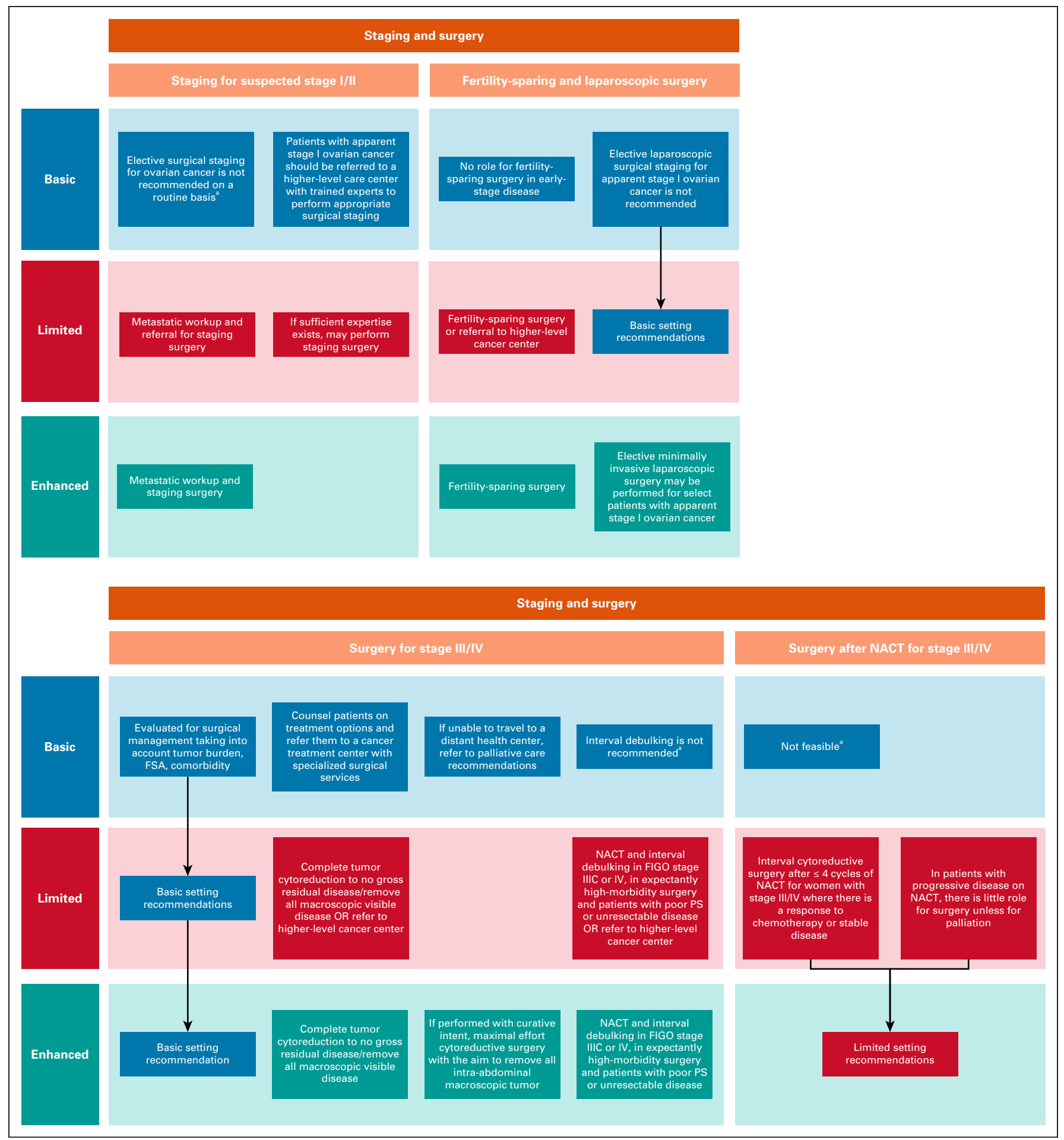

FIG A2. Staging and surgery recommendations by resource level. aBecause of current gaps in health system and human resource availability. FIGO, International Federation of Gynecology and Obstetrics; FSA, functional status assessment; NACT, neoadjuvant chemotherapy; PS, performance status. 


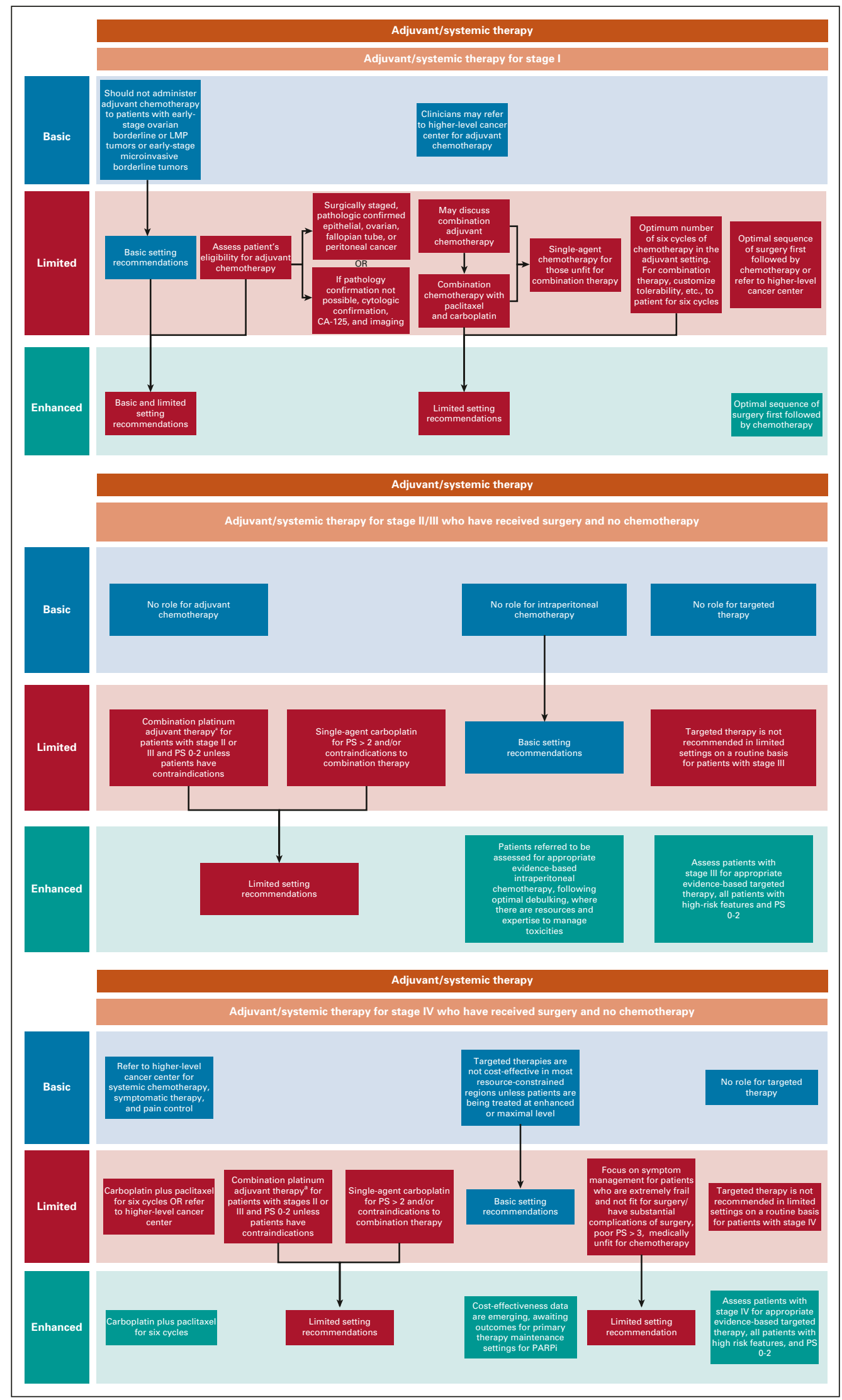

FIG A3. Adjuvant/systemic therapy recommendations by resource level. ${ }^{a}$ Clinicians should offer carboplatin plus paclitaxel (or single-agent carboplatin) once every 3 weeks for six cycles. CA-125, cancer antigen 125; LMP, low malignant potential; PARPi, poly (ADP-ribose) polymerase inhibitor; PS, performance status. 


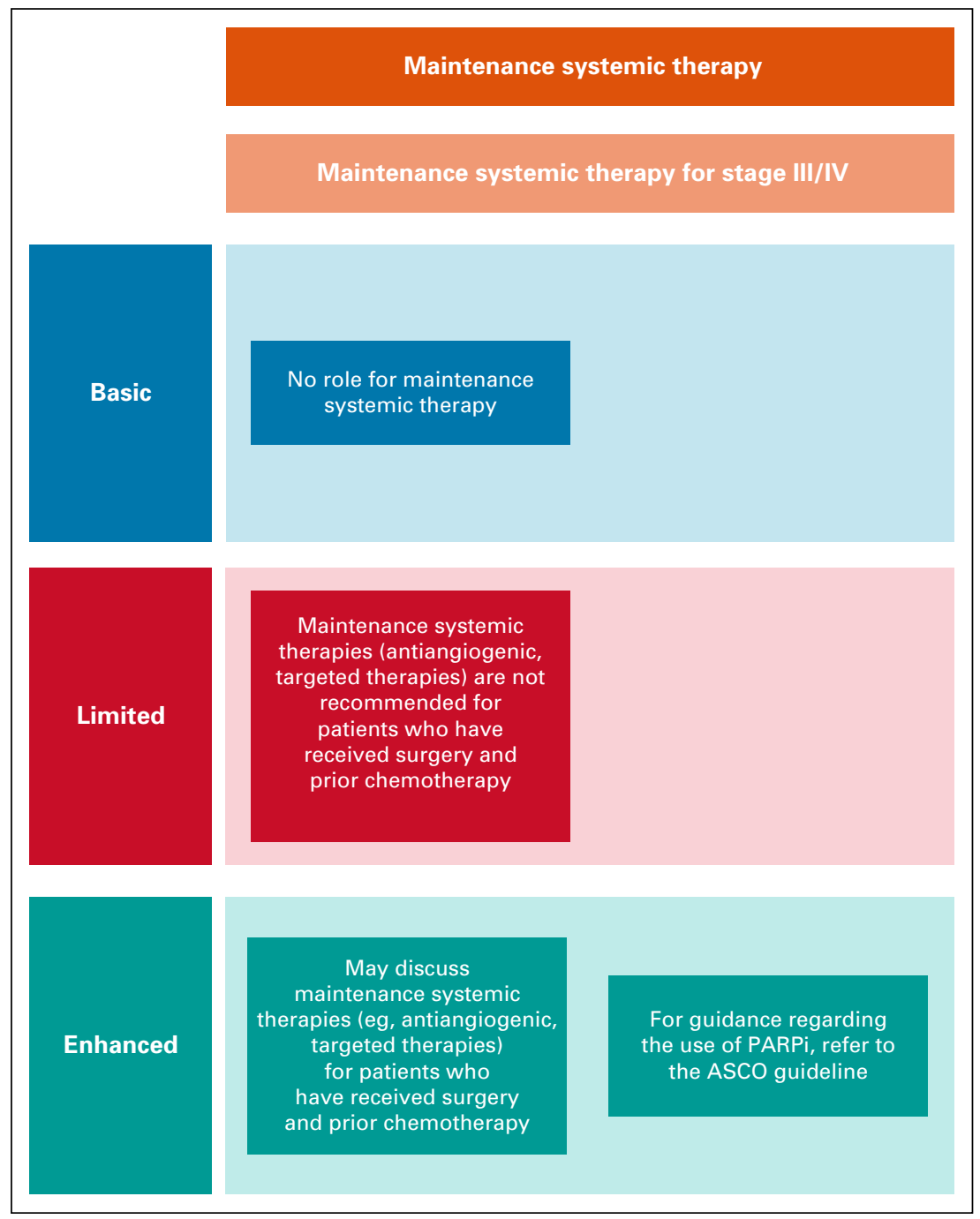

FIG A4. Maintenance systemic therapy recommendations by resource level. PARPi, poly (ADP-ribose) polymerase inhibitor. 


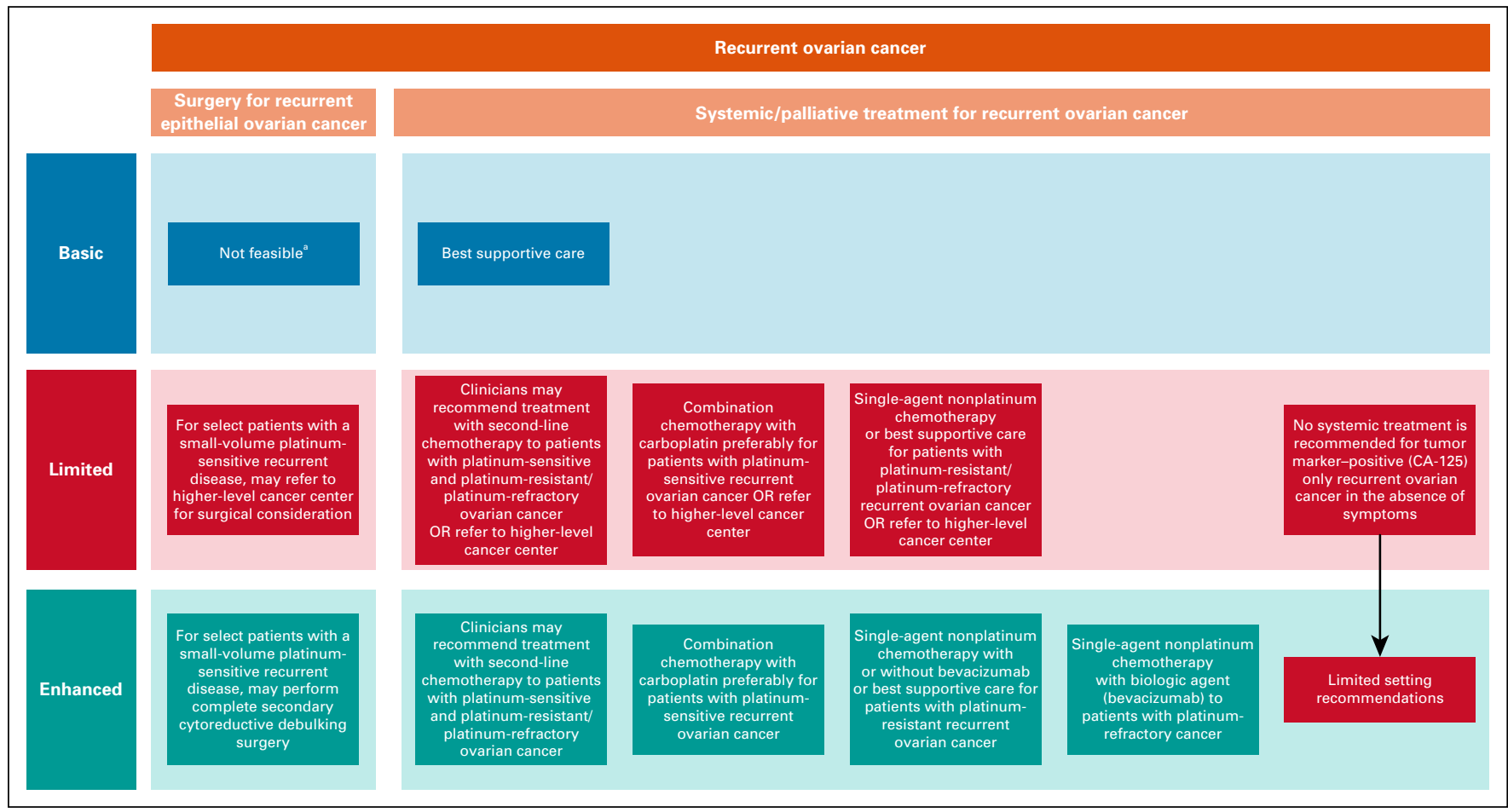

FIG A5. Recurrent ovarian cancer recommendations by resource level. ${ }^{2}$ Because of current gaps in health system and human resource availability. CA-125, cancer antigen 125. 


\section{Palliative care}

Palliative care for ovarian cancer

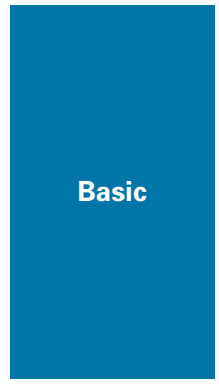

Clinicians should offer palliative care, including cancer pain and symptom

management, to all

patients diagnosed with

ovarian cancer

Early referral to palliative

care where available
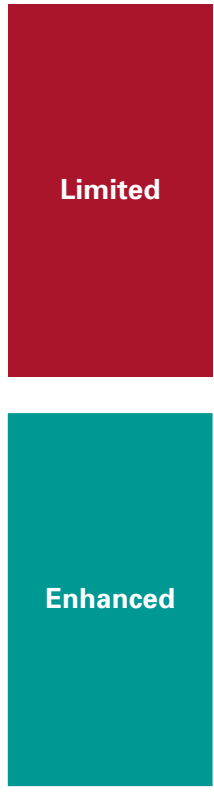

Basic setting recommendations
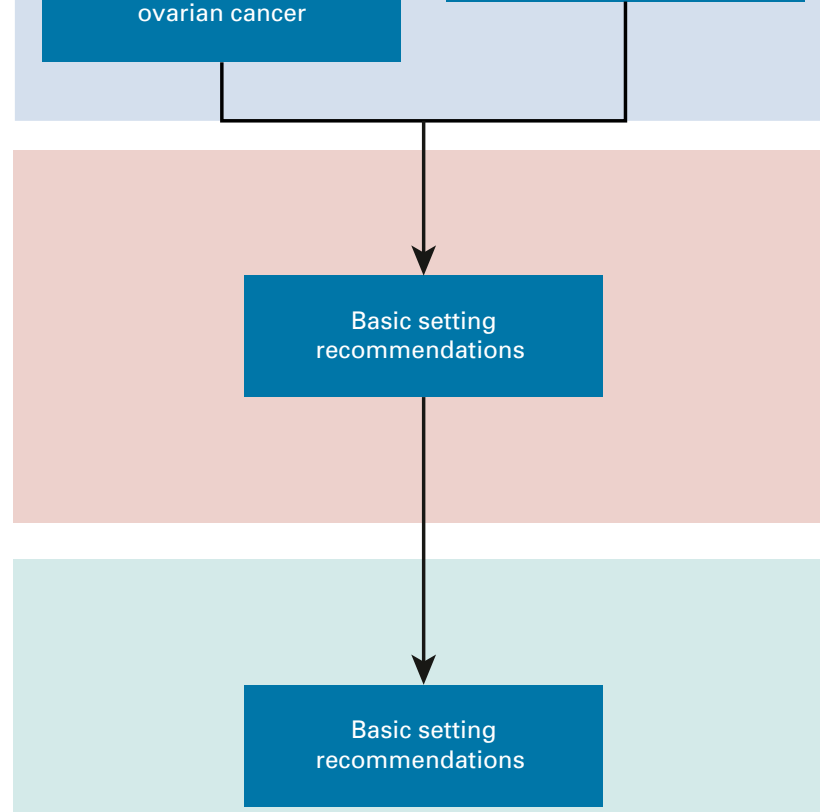

FIG A6. Palliative care recommendations by resource level. 


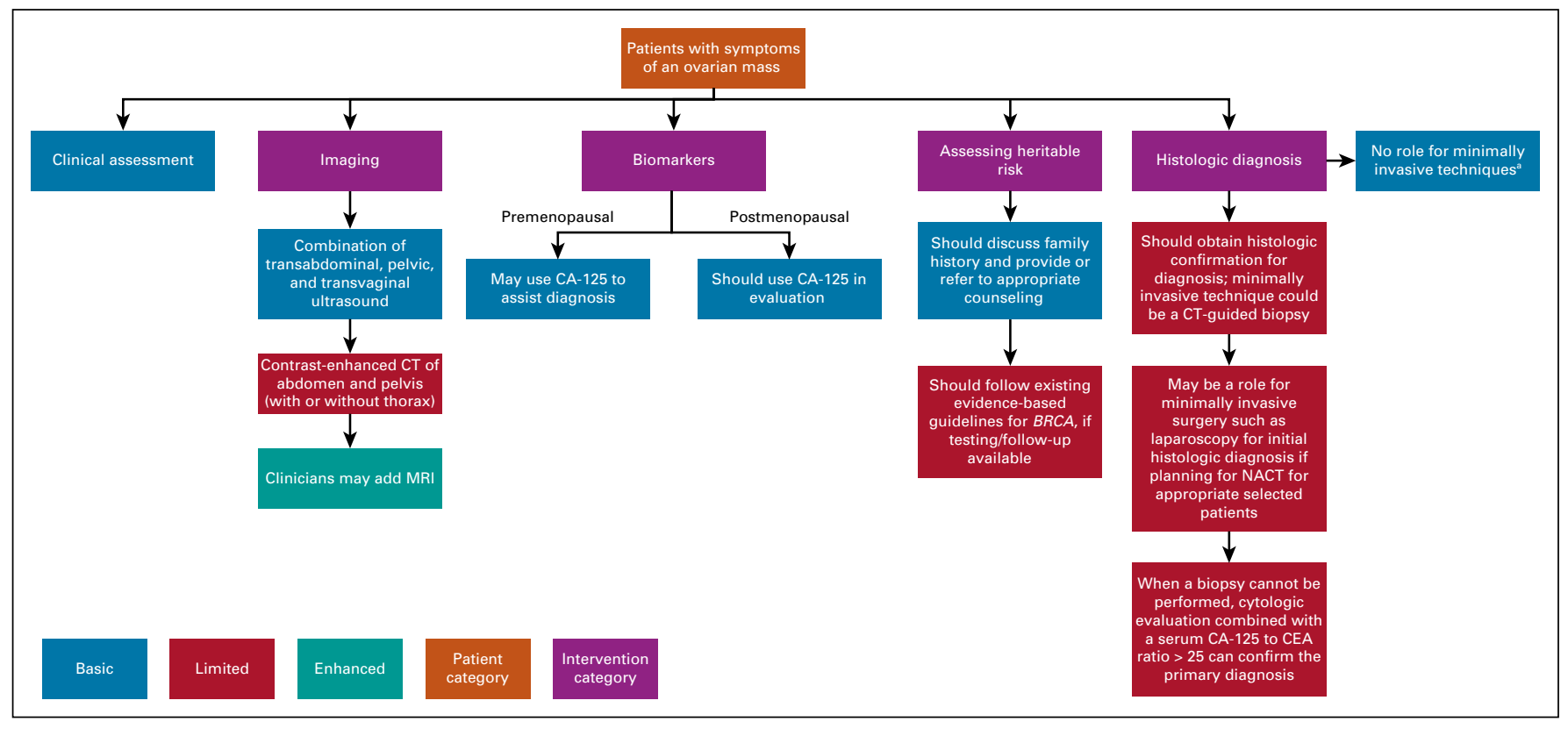

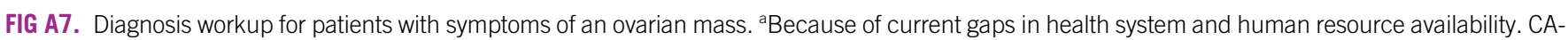
125, cancer antigen 125; CEA, carcinoembryonic antigen; CT, computed tomography; MRI, magnetic resonance imaging; NACT, neoadjuvant chemotherapy. 


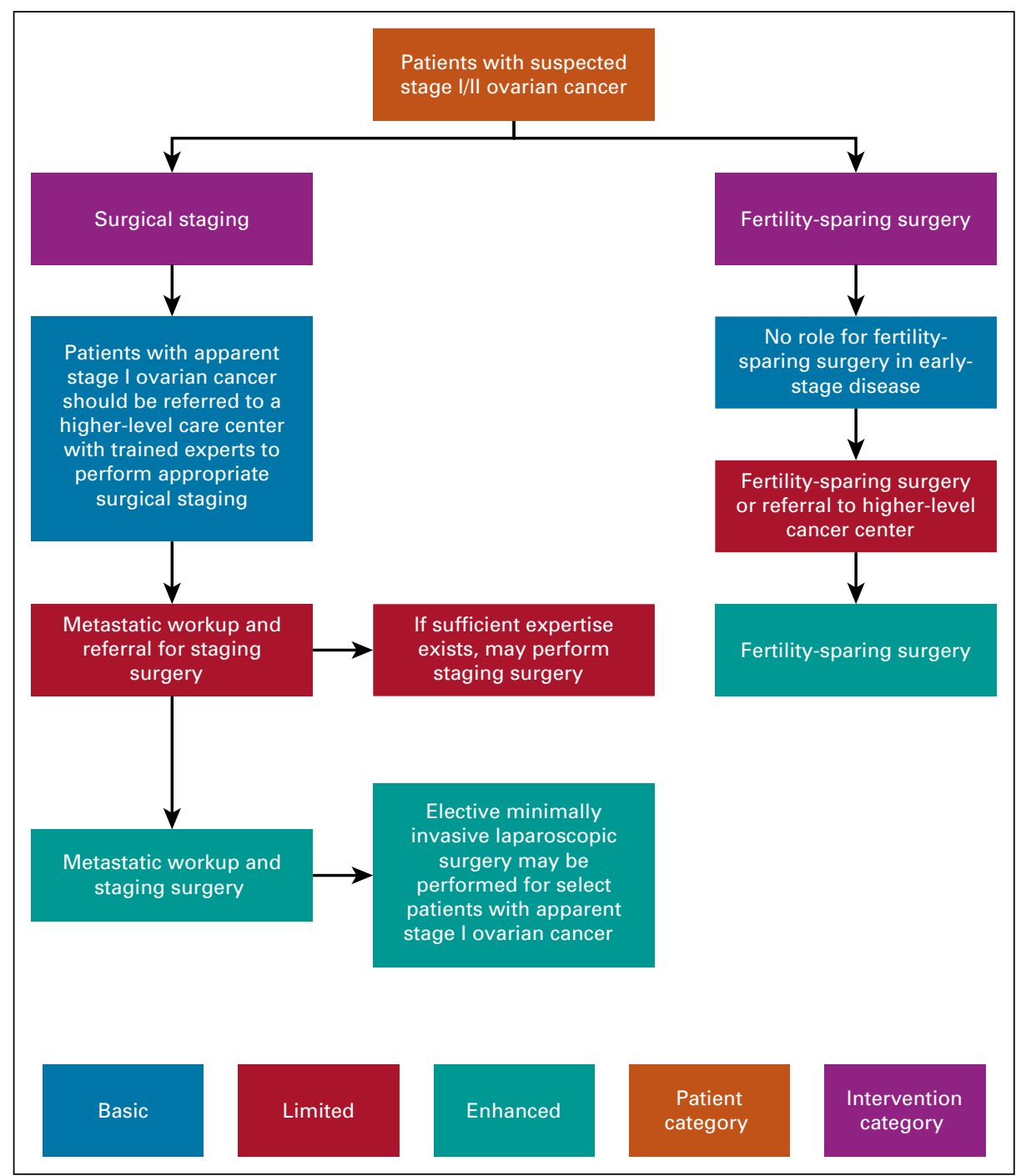

FIG A8. Surgery for patients with suspected stage I/II ovarian cancer. 


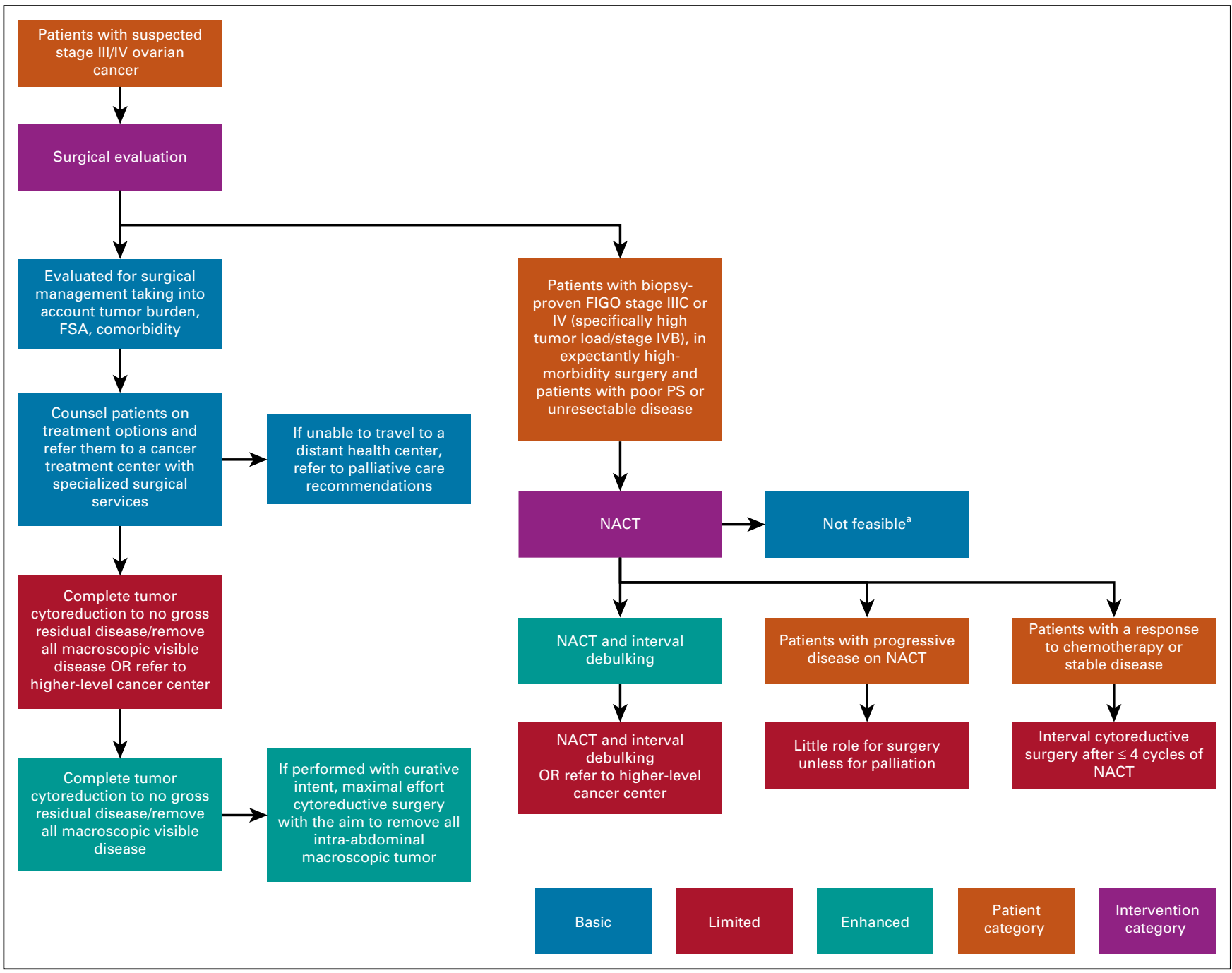

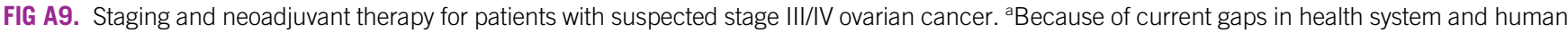
resource availability. FIGO, International Federation of Gynecology and Obstetrics; FSA, functional status assessment; NACT, neoadjuvant chemotherapy; PS, performance status. 


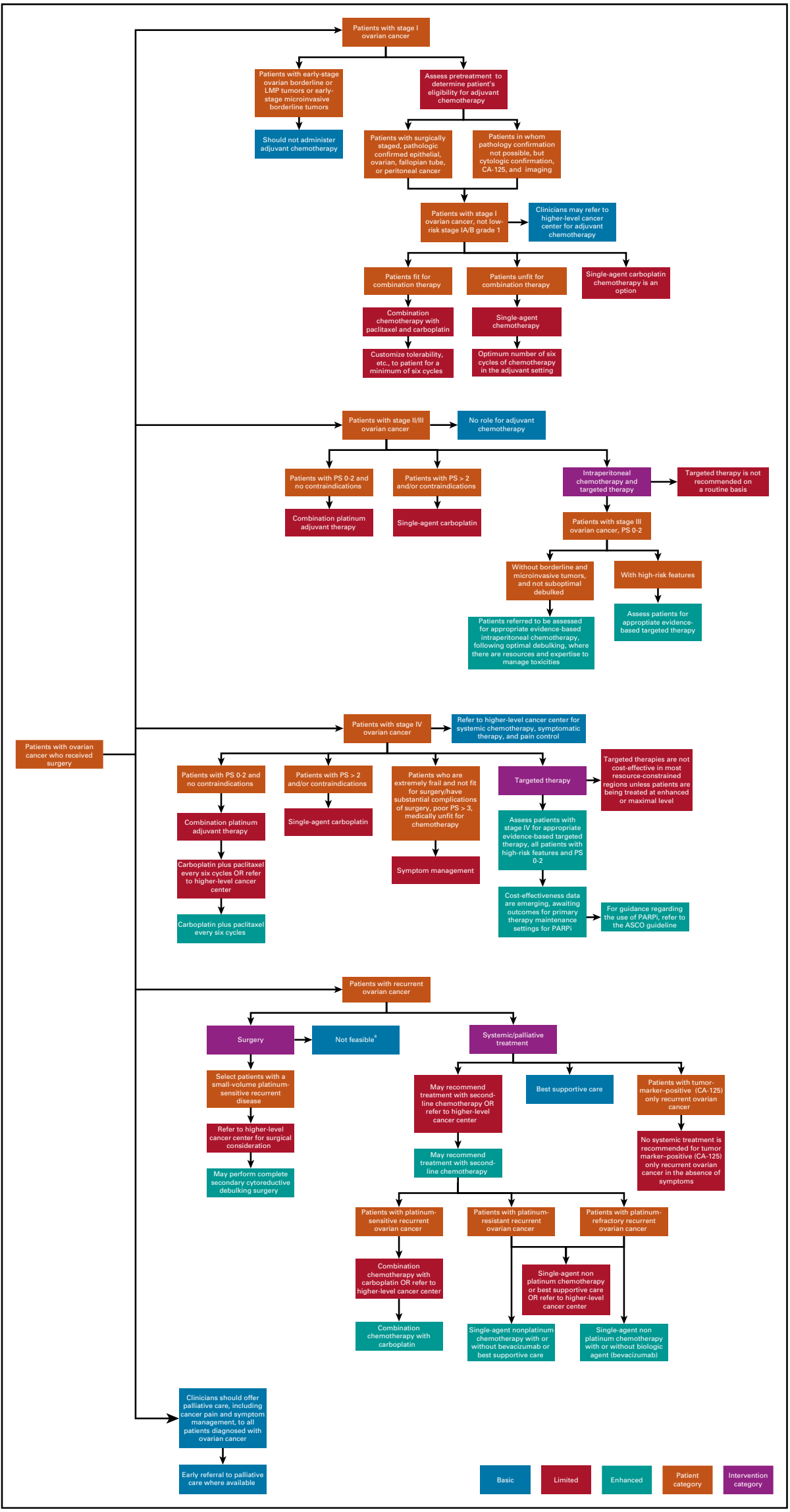


TABLE A1. Adapted Guidelines and Links

URL

\begin{tabular}{|c|c|c|}
\hline SIGN, ${ }^{12}$ 2013, Revised 2018 (Scotland) & $\begin{array}{l}\text { Management of epithelial ovarian cancer (SIGN } \\
\text { CPG 135)_-reviewed in } 2018\end{array}$ & $\begin{array}{l}\text { https://www.sign.ac.uk/sign-135-management-of- } \\
\text { epithelial-ovarian-cancer.html }\end{array}$ \\
\hline
\end{tabular}

Cancer Care Ontario, Toronto, ON, Program in Systemic therapy for recurrent ovarian cancer https://www.cancercareontario.ca/en/guidelinesEvidence-Based Care Guideline No.: 4-3v4. CCO PEBC4-3v4f, July 12, 2017. CCO. ${ }^{16}$

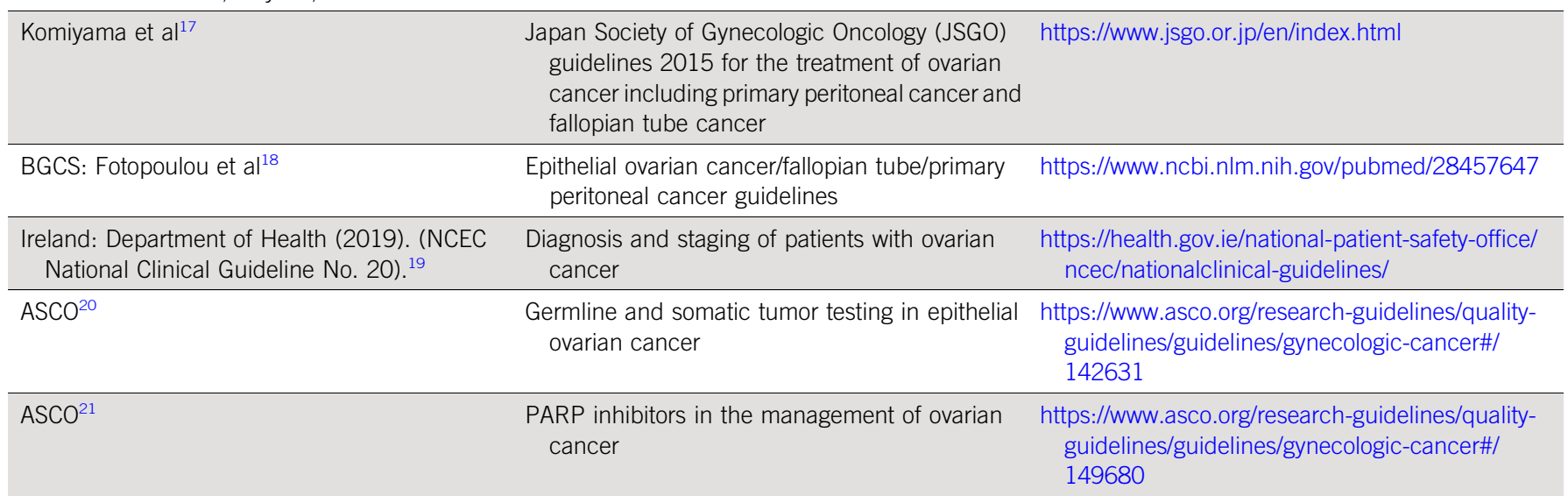

Abbreviations: BGCS, British Gynecological Cancer Society; CCO, Cancer Care Ontario; CPG, Clinical Practice Guideline; NACT, neoadjuvant chemotherapy; NCEC, National Clinical Effectiveness Committee; PARPi, poly (ADP-ribose) polymerase inhibitor; SGO, Society of Gynecologic Oncology; SIGN, Scottish Intercollegiate Guidelines Network.

FIG A10. Systemic therapy for patients with ovarian cancer (all stages). ${ }^{a}$ Because of current gaps in health system and human resource availability. CA-125, cancer antigen 125; LMP, low malignant potential; PARPi, poly (ADP-ribose) polymerase inhibitor; PS, performance status. 
TABLE A2. Assessment of Ovarian Masses and Treatment of Epithelial Ovarian Cancer: ASCO Resource-Stratified Guideline Expert Panel Membership

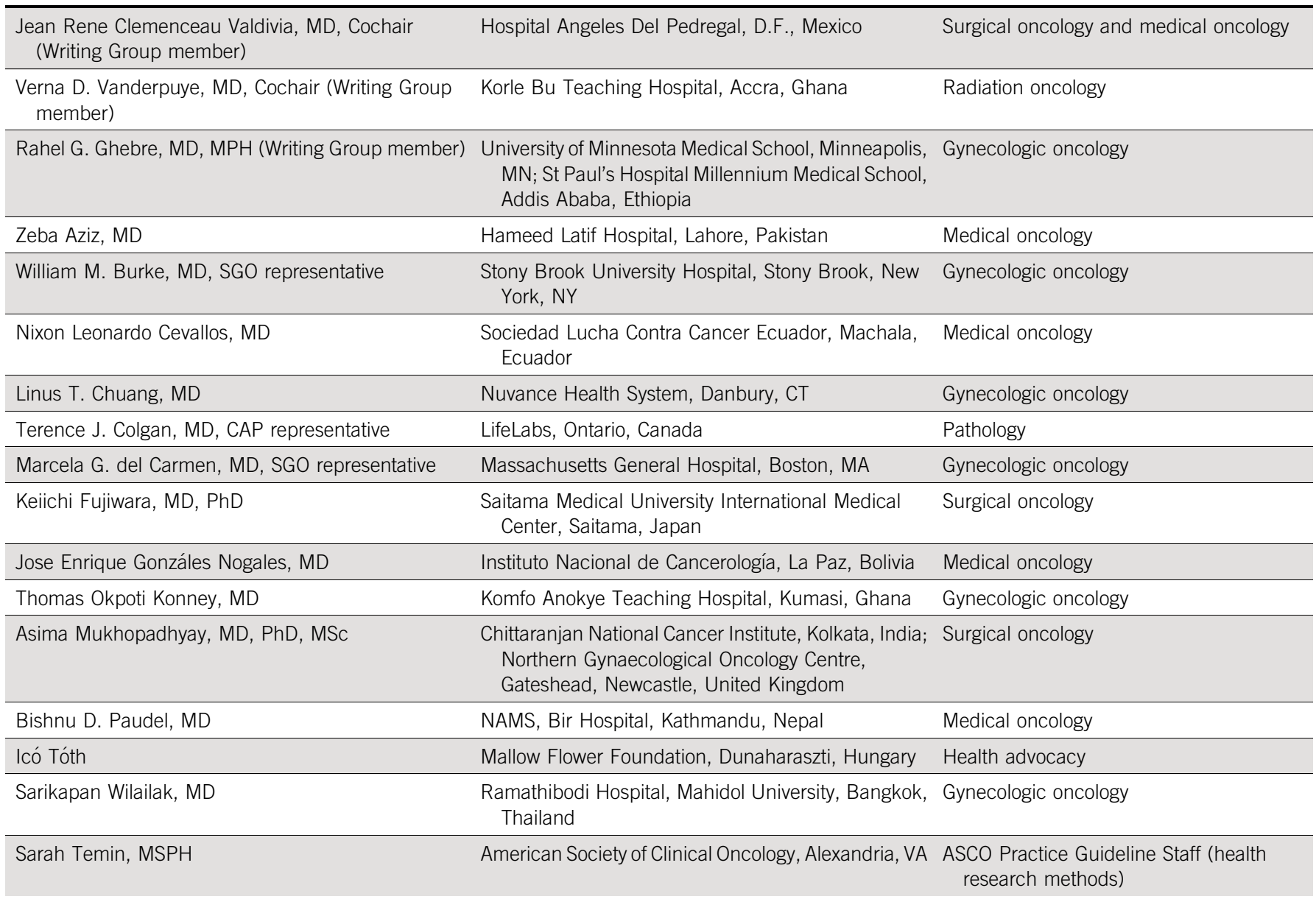

Abbreviations: CAP, College of American Pathology; SGO, Society of Gynecologic Oncology. 
TABLE A3. Assessment of Ovarian Masses and Treatment of Epithelial Ovarian Cancer: ASCO Resource-Stratified Guideline Consensus Panel Membership Name

Affiliation/Institution

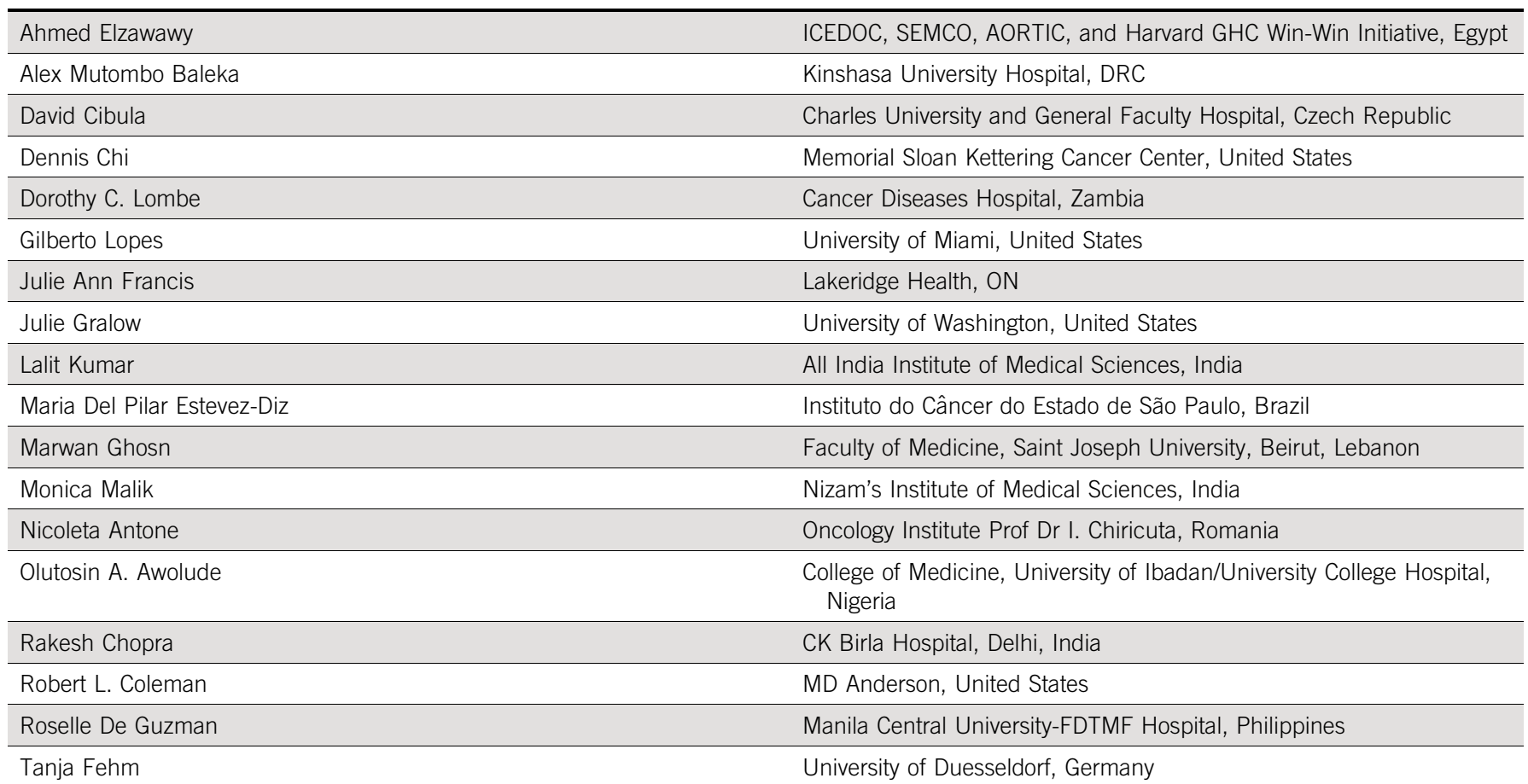

NOTE. Disclosures of potential conflicts of interest provided by the consensus panel members are available in the guideline supplement. 
TABLE A4. Brief Familial Risk Tools

Brief familial risk tools, see USPSTF (includes Ontario Family Health Assessment Tool, Manchester Scoring System, Referral Screening Tool, and Pedigree Assessment Tool)—included in USPSTF BRCA-Related Cancer: Risk

Assessment, Genetic Counseling, and Genetic Testing, August 20, 2019 [Ref

ID] https://www.uspreventiveservicestaskforce.org/uspstf/document/ RecommendationStatementFinal/brca-related-cancer-risk-assessmentgenetic-counseling-and-genetic-testing

Genetic testing guidelines/ASCO statements

USPSTF (screening) (BRCA) 2019

NCCN Clinical Practice Guidelines in Oncology (NCCN Guidelines) for Genetic/ Familial High-Risk Assessment: Breast, Ovarian, and Pancreatic. These NCCN Guidelines are currently available as Version 1.2020. December 14, 2019. nccn.org

\begin{tabular}{l} 
ASCO_policy statement ${ }^{25}$ \\
\hline ESMO \\
\hline ACOG \\
\hline SGO
\end{tabular}

Abbreviations: ACOG, American College of Obstetricians and Gynecologists; ESMO, European Society for Medical Oncology; NCCN, National Comprehensive Cancer Network; SGO, Society of Gynecologic Oncology; USPSTF, US Preventive Services Task Force. 